\title{
ADM-like Hamiltonian formulation of gravity in the teleparallel geometry: derivation of constraint algebra
}

\author{
Andrzej Okołów
}

Received: 11 June 2013 / Accepted: 4 November 2013 / Published online: 13 December 2013

(C) The Author(s) 2013. This article is published with open access at Springerlink.com

\begin{abstract}
We derive a new constraint algebra for a Hamiltonian formulation of the teleparallel equivalent of general relativity treated as a theory of cotetrad fields on a spacetime. The algebra turns out to be closed.
\end{abstract}

Keywords Teleparallel equivalent of general relativity $\cdot$ Hamiltonian formulation Constraint algebra

\section{Introduction}

In our previous paper [1] we presented a Hamiltonian formulation of the teleparallel equivalent of general relativity (TEGR) regarded as a theory of cotetrad fields on a spacetime - the formulation is meant to serve as a point of departure for canonical quantization à la Dirac of the theory (preliminary stages of the quantization are described in [2-4]). In [1] we found a phase space, a set of (primary and secondary) constraints on the phase space and a Hamiltonian. We also presented an algebra of the constraints. An important fact is that this algebra is closed i.e. a Poisson bracket of every pair of the constraints is a sum of all the constraints multiplied by some factors. This property of the constraint algebra together with a fact that the Hamiltonian is a sum of the constraints allowed us to conclude that (1) the set of constraints is complete and (2) all the constraints are of the first class.

Let us emphasize that knowledge of a complete set of constraints and their properties as well as knowledge of an explicite form of a constraint algebra is very important from the point of view the Dirac's approach to canonical quantization of constrained

\footnotetext{
A. Okołów (凶)

Institute of Theoretical Physics, Warsaw University, ul. Hoża 69, 00-681 Warsaw, Poland

e-mail: oko@fuw.edu.pl
} 
systems since the knowledge enables a right treatment of constraints in the procedure of quantization.

However, the derivation of the constraint algebra turned out to be too long to be included in [1]. To fill this gap, that is, to prove that the constraint algebra is correct we carry out the derivation in the present paper ${ }^{1}$. Moreover, to the best of our knowledge a derivation of a constraint algebra of TEGR treated as a theory of cotetrad fields has never been presented before-in papers [5,6] describing a distinct Hamiltonian formulation of this version ${ }^{2}$ of TEGR one can find a constraint algebra but its derivation is not shown.

The paper is organized as follows: in Sect. 2 we recall the description of the phase space and the constraints on it derived in [1]. In Sect. 3 we derive the constraint algebra. Section 4 contains a short summary.

Let us finally emphasize that since the present paper plays a role of an appendix to [1] we do not discuss here the results nor compare them to results of previous works - all these can be found in [1].

\section{Preliminaries}

Let $\mathbb{M}$ be a four-dimensional oriented vector space equipped with a scalar product $\eta$ of signature $(-,+,+,+)$. We fix an orthonormal basis $\left(v_{A}\right)(A=0,1,2,3)$ of $\mathbb{M}$ such that the components $\left(\eta_{A B}\right)$ of $\eta$ given by the basis form a matrix $\operatorname{diag}(-1,1,1,1)$. The matrix $\left(\eta_{A B}\right)$ and its inverse $\left(\eta^{A B}\right)$ will be used to, respectively, lower and raise capital Latin letter indices.

Let $\Sigma$ be a three-dimensional oriented manifold. We assume moreover that it is a compact manifold without boundary.

In [1] we obtained the phase space of TEGR as a Cartesian product of

1. a set of all quadruplets of one-forms $\left(\theta^{A}\right)(A=0,1,2,3)$ on $\Sigma$ such that for each quadruplet a metric

$$
q:=\eta_{A B} \theta^{A} \otimes \theta^{B}
$$

on $\Sigma$ is Riemannian (i.e. positive definite);

2. a set of all quadruplets $\left(p_{B}\right)(B=0,1,2,3)$ the two-form on $\Sigma$ - the two-form $p_{A}$ is the momentum conjugate to $\theta^{A}$.

The metric $q$ defines a volume form $\epsilon$ on $\Sigma$ and a Hodge operator $*$ acting on differential forms on the manifold. Throughout the paper we will often use functions on $\Sigma$ defined as follows [9]:

$$
\xi^{A}:=-\frac{1}{3 !} \varepsilon^{A}{ }_{B C D} *\left(\theta^{B} \wedge \theta^{C} \wedge \theta^{D}\right)
$$

\footnotetext{
${ }^{1}$ The derivation is also an example of an application of differential form calculus to a derivation of a constraint algebra which usually is done by means of tensor calculus.

2 There is another version of TEGR configuration variables of which are cotetrad fields and flat Lorentz connections of non-zero torsion. For a complete Hamiltonian analysis of this version of TEGR see [7].
} 
where $\varepsilon_{A B C D}$ are components of a volume form on $\mathbb{M}$ given by the scalar product $\eta$.

Components of $q$ in a local coordinate frame $\left(x^{i}\right)(i=1,2,3)$ on $\Sigma$ will be denoted by $q_{i j}$. Obviously

$$
q_{i j}=\eta_{A B} \theta_{i}^{A} \theta_{j}^{B}
$$

where $\theta_{i}^{A}$ are components of $\theta^{A}$. The metric $q$ and its inverse $q^{-1}$,

$$
q^{-1}:=q^{i j} \partial_{i} \otimes \partial_{j}, \quad q^{i j} q_{j k}=\delta_{k}^{i},
$$

will be used to, respectively, lower and raise, indices (here: lower case Latin letters) of components of tensor fields defined on $\Sigma$. In particular we will often map one-forms to vector fields on $\Sigma$ by means of $q$-a vector field corresponding to a one form $\alpha$ will be denoted by $\vec{\alpha}$ i.e. if $\alpha=\alpha_{i} d x^{i}$ then

$$
\vec{\alpha}:=q^{i j} \alpha_{i} \partial_{j}
$$

Let us emphasize that all object defined by $q$ (as $\epsilon, *, \xi^{A}$ and $q^{-1}$ ) are functions of $\left(\theta^{A}\right)$ which means that they are functions on the phase space.

In [1] we found some constraints on the phase space of TEGR. Smeared versions of the constraints read

$$
\begin{aligned}
B(a):= & \int_{\Sigma} a \wedge\left(\theta^{A} \wedge * d \theta_{A}+\xi^{A} p_{A}\right), \\
R(b):= & \int_{\Sigma} b \wedge\left(\theta^{A} \wedge * p_{A}-\xi^{A} d \theta_{A}\right), \\
S(M):= & \int_{\Sigma} M\left(\frac{1}{2}\left(p_{A} \wedge \theta^{B}\right) \wedge *\left(p_{B} \wedge \theta^{A}\right)-\frac{1}{4}\left(p_{A} \wedge \theta^{A}\right) \wedge *\left(p_{B} \wedge \theta^{B}\right)\right. \\
& -\xi^{A} \wedge d p_{A}+\frac{1}{2}\left(d \theta_{A} \wedge \theta^{B}\right) \wedge *\left(d \theta_{B} \wedge \theta^{A}\right) \\
& \left.-\frac{1}{4}\left(d \theta_{A} \wedge \theta^{A}\right) \wedge *\left(d \theta_{B} \wedge \theta^{B}\right)\right), \\
V(\vec{M}):= & \left.\left.\int_{\Sigma}-d \theta^{A} \wedge(\vec{M}\lrcorner p_{A}\right)-(\vec{M}\lrcorner \theta^{A}\right) \wedge d p_{A},
\end{aligned}
$$

where $a, b, M$ and $\vec{M}$ are smearing fields on $\Sigma: a$ and $b$ are one-forms, $M$ is a function and $\vec{M}$ a vector field on the manifold. The smearing field possess altogether ten degrees of freedom per point of $\Sigma$. In [1] we called $B(a)$ boost constraint and $R(b)$ rotation constraint. $S(M)$ is a scalar constraint and $V(\vec{M})$ a vector constraint of TEGR. 


\section{Derivation of the constraint algebra}

In this section we will calculate Poisson brackets of the constraints presented above and show that each Poisson bracket is a sum of the constraints smeared with some fields. We assume that the reader is familiar with tensor calculus, differential form calculus (including the contraction $\vec{X}\lrcorner \alpha$ of a vector field $\vec{X}$ with a differential form $\alpha$ ) and properties of a Hodge operator on a three-dimensional manifold defined by a Riemannian metric.

\subsection{Preliminaries}

\subsubsection{Poisson bracket}

If $F$ and $G$ are functionals on the phase space then their Poisson bracket [8]

$$
\{F, G\}=\int_{\Sigma}\left(\frac{\delta F}{\delta \theta^{A}} \wedge \frac{\delta G}{\delta p_{A}}-\frac{\delta G}{\delta \theta^{A}} \wedge \frac{\delta F}{\delta p_{A}}\right),
$$

where the functional derivatives with respect to $\theta^{A}$ and $p_{A}$ are defined as follows [9]: $\delta F / \delta \theta^{A}$ is a differential two-form on $\Sigma$ and $\delta F / \delta p_{A}$ is a differential one-form on $\Sigma$ such that

$$
\delta F=\int_{\Sigma} \delta \theta^{A} \wedge \frac{\delta F}{\delta \theta^{A}}+\delta p_{A} \wedge \frac{\delta F}{\delta p_{A}}
$$

for every $\delta \theta^{A}$ and $\delta p_{A}$.

Calculating functional derivatives of the smeared constraints would be straightforward if (1) the Hodge operator $*$ did not depend on $\theta^{A}$ and (2) the constraints did not depend on $\xi^{A}$ being a complicated function of $\theta^{A}$. Thus explicite formulae describing these derivatives are needed.

Given $k$-forms $\alpha$ and $\beta$, denote

$$
\left.\left.\left.\left.\left.\alpha *_{A}^{\prime} \beta \equiv \vec{\theta}^{B}\right\lrcorner\left[\eta_{A B} \alpha \wedge * \beta-\left(\vec{\theta}_{A}\right\lrcorner \alpha\right) \wedge *\left(\vec{\theta}_{B}\right\lrcorner \beta\right)-\left(\vec{\theta}_{B}\right\lrcorner \alpha\right) \wedge *\left(\vec{\theta}_{A}\right\lrcorner \beta\right)\right] .
$$

If the forms $\alpha, \beta$ do not depend on the canonical variables then [9]

$$
\frac{\delta}{\delta \theta^{A}} \int_{\Sigma} \alpha \wedge * \beta=\alpha *_{A}^{\prime} \beta
$$

An important property of every two-form $\alpha *_{A}^{\prime} \beta$ is that it vanishes once contracted with the function $\xi^{A}[9]$ :

$$
\xi^{A}\left(\alpha *_{A}^{\prime} \beta\right)=0 \text {. }
$$


Consider now a three-form $\kappa_{A}$ on $\Sigma$ which does not depend on $\theta^{A}$ and $p_{A}$ and a functional

$$
F=\int_{\Sigma} \xi^{D} \kappa_{D}=\int_{\Sigma}\left(* \xi^{D}\right) \wedge *\left(\kappa_{D}\right)=\int_{\Sigma}-\frac{1}{3 !} \varepsilon^{D}{ }_{B C A} \theta^{B} \wedge \theta^{C} \wedge \theta^{A} \wedge * \kappa_{D}
$$

Using (3.2) we obtain

$$
\frac{\delta F}{\delta \theta^{A}}=-\frac{1}{2} \varepsilon^{D}{ }_{B C A} \theta^{B} \wedge \theta^{C} \wedge * \kappa_{D}+\left(* \xi^{D}\right) *_{A}^{\prime} \kappa_{D}
$$

\subsubsection{Auxiliary formulae}

Auxiliary formulae presented below will be used throughout the calculations. Except them we will need many other formulae which will be derived in the subsequent subsections.

The functions $\left(\xi^{A}\right)$ satisfy the following important conditions [10]:

$$
\begin{aligned}
& \xi^{A} \xi_{A}=-1, \quad \xi^{A} \theta_{A}=0, \\
& \xi^{A} d \xi_{A}=0, \quad d \xi^{A} \wedge \theta_{A}+\xi^{A} d \theta_{A}=0 .
\end{aligned}
$$

These formulae will be used very often and therefore it would be troublesome to refer to them each time. Therefore we kindly ask the reader to keep the formulae in mind since they will be used without any reference.

For any one-form $\alpha$ and any $k$-form $\beta$ [9]

$$
*(* \beta \wedge \alpha)=\vec{\alpha}\lrcorner \beta
$$

Setting $\beta=* \gamma$ and taking into account that $* *=\mathrm{id}$ we obtain an identity

$$
*(\gamma \wedge \alpha)=\vec{\alpha}\lrcorner(* \gamma)
$$

valid for every $l$-form $\gamma$.

It was shown in [1] that

$$
\begin{aligned}
\left.\vec{\theta}^{B}\right\lrcorner \theta^{A} & =\eta^{A B}+\xi^{A} \xi^{B}, \\
\left.\theta^{A} \wedge\left(\vec{\theta}_{A}\right\lrcorner \alpha\right) & =k \alpha
\end{aligned}
$$

where $\alpha$ is a $k$-form on $\Sigma$.

\subsubsection{Tensor calculus}

Although our original wish was to carry out all necessary calculations using differential form calculus only we were forced in some cases to use tensor calculus. Below we gathered expressions very useful for the calculations. 
Let $\nabla$ denote a covariant derivative on $\Sigma$ defined by the Levi-Civita connection given by the metric $q$. Then

$$
\begin{gathered}
0=\nabla_{i} q_{j k}=\nabla_{i}\left(\theta_{A j} \theta_{k}^{A}\right)=\left(\nabla_{i} \theta_{A j}\right) \theta_{k}^{A}+\theta_{A j}\left(\nabla_{i} \theta_{k}^{A}\right), \\
\left(\nabla_{a} \theta^{B b}\right) \theta_{B b}=0, \quad \nabla_{a} \epsilon_{i j k}=0
\end{gathered}
$$

(the first equation above holds by virtue of (2.3)).

For any one-forms $\alpha$ and $\beta$

$$
\begin{aligned}
d \alpha & =\nabla_{a} \alpha_{b} d x^{a} \wedge d x^{b}, \quad * d \alpha=\left(\nabla_{a} \alpha_{b}\right) \epsilon_{c}^{a b} d x^{c} \\
d \alpha \wedge \beta & =\left(\nabla_{a} \alpha_{b}\right) \beta_{c} d x^{a} \wedge d x^{b} \wedge d x^{c}, \quad *(d \alpha \wedge \beta)=\left(\nabla_{a} \alpha_{b}\right) \beta_{c} \epsilon^{a b c} .
\end{aligned}
$$

If $\alpha$ is a one-form, $\beta$ a two-form and $\gamma$ a three-form then

$$
\begin{aligned}
& * d * \alpha=q^{a b} \nabla_{a} \alpha_{b}=\nabla^{a} \alpha_{a}, \quad * d * \beta=q^{a b} \nabla_{a} \beta_{c b} d x^{c}=\nabla^{b} \beta_{c b} d x^{c}, \\
& * d * \gamma=q^{a b} \nabla_{a} \gamma_{d c b} d x^{d} \otimes d x^{c}=\nabla^{b} \gamma_{d c b} d x^{d} \otimes d x^{c}=\frac{1}{2} \nabla^{b} \gamma_{d c b} d x^{d} \wedge d x^{c} .
\end{aligned}
$$

We will also apply the following identities (for a proof see e.g. [9]):

$$
\epsilon_{i b c} \epsilon^{a b c}=2 \delta^{a}{ }_{i}, \quad \epsilon_{i j c} \epsilon^{a b c}=2 \delta^{[a}{ }_{i} \delta^{b]}{ }_{j}, \quad \epsilon_{i j k} \epsilon^{a b c}=3 ! \delta^{[a}{ }_{i} \delta^{b}{ }_{j} \delta^{c]}{ }_{k} .
$$

and a formula

$$
\alpha \wedge * \beta=\frac{1}{k !} \alpha_{a_{1} \ldots a_{k}} \beta^{a_{1} \ldots a_{k}} \epsilon
$$

where $\alpha$ and $\beta$ are $k$-forms.

\subsection{Poisson brackets of $B(a)$ and $R(b)$}

\subsubsection{Auxiliary formulae}

The following formulae are useful while calculating the brackets:

$$
\begin{aligned}
b \wedge \alpha \wedge *\left(b^{\prime} \wedge \beta\right)-\left(b \leftrightarrow b^{\prime}\right) & =*\left(b \wedge b^{\prime}\right) \wedge \alpha \wedge \beta, \\
\left(\alpha *_{A}^{\prime} \beta\right) \wedge *\left(b \wedge \theta^{A}\right) & =0, \\
\theta_{A} \wedge *\left(\alpha \wedge \theta^{A}\right) & =(3-k) * \alpha, \\
\epsilon_{D B C A} \theta^{B} \wedge \theta^{C} \wedge *\left(b \wedge \theta^{A}\right) & =0, \\
-\frac{1}{2} \epsilon^{D}{ }_{B C A} \theta^{B} \wedge \theta^{C} \xi^{A} & =* \theta^{D} .
\end{aligned}
$$

In (3.14) $\alpha, \beta, b, b^{\prime}$ are one-forms, in (3.15) $\alpha$ and $\beta$ are $k$-forms, and $b$ is a one-form, in (3.16) $\alpha$ is a $k$-form, and in (3.17) $b$ is a one-form. 
Proof of (3.14)

$$
\begin{aligned}
b \wedge \alpha \wedge *\left(b^{\prime} \wedge \beta\right)-\left(b \leftrightarrow b^{\prime}\right) & =b \wedge * *\left[\alpha \wedge *\left(b^{\prime} \wedge \beta\right)\right]-\left(b \leftrightarrow b^{\prime}\right) \\
& \left.\left.=-b \wedge *\left[(\vec{\alpha}\lrcorner b^{\prime}\right) \beta-b^{\prime} \vec{\alpha}\right\lrcorner \beta\right]-\left(b \leftrightarrow b^{\prime}\right) \\
& \left.=\vec{\alpha}\lrcorner\left(b \wedge b^{\prime}\right) \wedge * \beta=-\left(b \wedge b^{\prime}\right) \vec{\alpha}\right\lrcorner * \beta \\
& =\left(b \wedge b^{\prime}\right) \wedge *(\alpha \wedge \beta)=*\left(b \wedge b^{\prime}\right) \wedge(\alpha \wedge \beta),
\end{aligned}
$$

where in the second step we used (3.5), in the third the fact that $b \wedge * b^{\prime}$ is symmetric in $b$ and $b^{\prime}$ and finally in the fifth step we applied (3.6).

Proof of (3.15) To prove (3.15) note that the two-form $\alpha *_{A}^{\prime} \beta$ given by (3.1) is of the form $\left.\vec{\theta}^{B}\right\lrcorner \gamma_{A B}$, where the three-form $\gamma_{A B}$ is symmetric in $A$ and $B: \gamma_{A B}=\gamma_{B A}$. Thus

$$
\begin{aligned}
\left(\alpha *_{A}^{\prime} \beta\right) \wedge *\left(b \wedge \theta^{A}\right) & \left.=\left(\vec{\theta}^{B}\right\lrcorner \gamma_{A B}\right) \wedge *\left(b \wedge \theta^{A}\right) \\
\left.=\gamma_{A B} \wedge \vec{\theta}^{B}\right\lrcorner *\left(b \wedge \theta^{A}\right) & =\gamma_{A B} \wedge *\left(b \wedge \theta^{A} \wedge \theta^{B}\right),
\end{aligned}
$$

where in the last step we used (3.6). But $\left(b \wedge \theta^{A} \wedge \theta^{B}\right)$ is antisymmetric in $A$ and $B$, hence (3.15) follows.

Proof of (3.16)

$$
\left.\theta_{A} \wedge *\left(\alpha \wedge \theta^{A}\right)=\theta_{A} \wedge \vec{\theta}^{A}\right\lrcorner * \alpha=(3-k) * \alpha,
$$

where in the first step we used (3.6) and in the second one we applied (3.8).

Proof of (3.17) Let us transform the following expression by means of (3.6):

$$
\begin{aligned}
\theta^{B} \wedge \theta^{C} \wedge *\left(b \wedge \theta^{A}\right) & \left.=\theta^{B} \wedge \theta^{C} \wedge \vec{\theta}^{A}\right\lrcorner * b \\
& \left.\left.=-\left(\vec{\theta}^{A}\right\lrcorner \theta^{B}\right) \wedge \theta^{C} \wedge * b+\theta^{B}\left(\vec{\theta}^{A}\right\lrcorner \theta^{C}\right) \wedge * b .
\end{aligned}
$$

The first term at the r.h.s. of this equation is symmetric in $A$ and $B$, while the second one-in $A$ and $C$. This means that both terms vanish once contracted with $\epsilon_{D B C A}$.

The last formula (3.18) is proven in [9].

\subsubsection{Poisson bracket $\left\{R(b), R\left(b^{\prime}\right)\right\}$}

Let us define

$$
R_{1}(b):=\int_{\Sigma} b \wedge \theta^{A} \wedge * p_{A}, \quad R_{2}(b):=\int_{\Sigma} b \wedge \xi^{A} d \theta_{A} .
$$


Then by virtue of (2.6) $R(b)=R_{1}(b)-R_{2}(b)$. Corresponding variational derivatives read

$$
\begin{aligned}
& \frac{\delta R_{1}}{\delta \theta^{A}}=-b \wedge * p_{A}+\left(b \wedge \theta^{B}\right) *_{A}^{\prime} p_{B}, \quad \frac{\delta R_{1}}{\delta p_{A}}=*\left(b \wedge \theta^{A}\right), \\
& \frac{\delta R_{2}}{\delta \theta^{A}}=d\left(b \xi_{A}\right)+\left(b \wedge d \theta^{B}\right) *_{A}^{\prime}\left(* \xi_{B}\right)-\frac{1}{2} \epsilon_{D B C A} \theta^{B} \wedge \theta^{C} *\left(b \wedge d \theta^{D}\right), \quad \frac{\delta R_{2}}{\delta p_{A}}=0 .
\end{aligned}
$$

Due to (3.15)

$\left\{R_{1}(b), R_{1}\left(b^{\prime}\right)\right\}=\int_{\Sigma}-b \wedge * p_{A} \wedge *\left(b^{\prime} \wedge \theta^{A}\right)-\left(b \leftrightarrow b^{\prime}\right)=\int_{\Sigma} *\left(b \wedge b^{\prime}\right) \wedge \theta^{A} \wedge * p_{A}$,

where in the last step we used (3.14). Similarly, using (3.15) we obtain

$$
\begin{aligned}
& \left\{R_{1}(b), R_{2}\left(b^{\prime}\right)\right\}-\left\{R_{1}\left(b^{\prime}\right), R_{2}(b)\right\} \\
& =\int_{\Sigma}\left[-d\left(b^{\prime} \xi_{A}\right)+\frac{1}{2} \epsilon_{D B C A} \theta^{B} \wedge \theta^{C} *\left(b^{\prime} \wedge d \theta^{D}\right)\right] \wedge *\left(b \wedge \theta^{A}\right)-\left(b \leftrightarrow b^{\prime}\right) \\
& =\int_{\Sigma}\left[b^{\prime} \wedge d \xi^{A} \wedge *\left(b \wedge \theta^{A}\right)+\frac{1}{2} \epsilon_{D B C A} \theta^{B} \wedge \theta^{C} \wedge *\left(b \wedge \theta^{A}\right) *\left(b^{\prime} \wedge d \theta^{D}\right)\right]-\left(b \leftrightarrow b^{\prime}\right) .
\end{aligned}
$$

Note now that due to (3.17) the term containing $\epsilon_{D B C A}$ vanishes. By virtue of (3.14)

$$
\left\{R_{1}(b), R_{2}\left(b^{\prime}\right)\right\}-\left\{R_{1}\left(b^{\prime}\right), R_{2}(b)\right\}=\int_{\Sigma} *\left(b \wedge b^{\prime}\right) \wedge \xi^{A} d \theta_{A}
$$

Because $\left\{R_{2}(b), R_{2}\left(b^{\prime}\right)\right\}=0$ we finally obtain

$$
\left\{R(b), R\left(b^{\prime}\right)\right\}=\int_{\Sigma} *\left(b \wedge b^{\prime}\right) \wedge\left(\theta^{A} \wedge * p_{A}-\xi^{A} d \theta_{A}\right)=R\left(*\left(b \wedge b^{\prime}\right)\right) .
$$

\subsubsection{Poisson brackets $\left\{B(a), B\left(a^{\prime}\right)\right\}$ and $\{B(a), R(b)\}$}

In a similar way using the formulae (3.3), (3.5), (3.14) and (3.18) we obtain

$$
\left\{B(a), B\left(a^{\prime}\right)\right\}=-\int_{\Sigma} *\left(a \wedge a^{\prime}\right) \wedge\left(\theta^{A} \wedge * p_{A}-\xi^{A} d \theta_{A}\right)=-R\left(*\left(a \wedge a^{\prime}\right)\right) .
$$


To calculate $\{B(a), R(b)\}$ we use the formulae (3.3) and (3.14)-(3.18) obtaining

$$
\begin{aligned}
\{B(a), R(b)\}= & B(*(a \wedge b))+\int_{\Sigma}-b \wedge * d \theta_{A} \wedge *\left(a \wedge \theta^{A}\right) \\
& +\left[* * d *\left(a \wedge \theta_{A}\right)+* d *\left(\theta_{A} \wedge * a\right)\right] \wedge *\left(b \wedge \theta^{A}\right) .
\end{aligned}
$$

Using tensor calculus (Sect. 3.1.3) one can show that the last line above is zero hence

$$
\{B(a), R(b)\}=B(*(a \wedge b)) .
$$

\subsection{Poisson bracket of $S(M)$ and $S\left(M^{\prime}\right)$}

Following [9] we split the constraint $S(M)$ given by (2.7) into three functionals

$$
\begin{aligned}
& S_{1}(M):=\int_{\Sigma} M\left[\frac{1}{2}\left(p_{A} \wedge \theta^{B}\right) \wedge *\left(p_{B} \wedge \theta^{A}\right)-\frac{1}{4}\left(p_{A} \wedge \theta^{A}\right) \wedge *\left(p_{B} \wedge \theta^{B}\right)\right] \\
& S_{2}(M):=-\int_{\Sigma} M \xi^{A} d p_{A} \\
& S_{3}(M):=\int_{\Sigma} M\left[\frac{1}{2}\left(d \theta_{A} \wedge \theta^{B}\right) \wedge *\left(d \theta_{B} \wedge \theta^{A}\right)-\frac{1}{4}\left(d \theta_{A} \wedge \theta^{A}\right) \wedge *\left(d \theta_{B} \wedge \theta^{B}\right)\right] .
\end{aligned}
$$

\subsubsection{Poisson brackets $\left\{S_{i}(M), S_{j}\left(M^{\prime}\right)\right\}$}

Functional derivatives of the functionals read:

$$
\begin{aligned}
\frac{\delta S_{1}}{\delta \theta^{A}}= & M\left(p_{B} *\left(p_{A} \wedge \theta^{B}\right)-\frac{1}{2} p_{A} *\left(p_{B} \wedge \theta^{B}\right)\right. \\
& \left.+\frac{1}{2}\left(p_{C} \wedge \theta^{B}\right) *_{A}^{\prime}\left(p_{B} \wedge \theta^{C}\right)-\frac{1}{4}\left(p_{B} \wedge \theta^{B}\right) *_{A}^{\prime}\left(p_{C} \wedge \theta^{C}\right)\right), \\
\frac{\delta S_{1}}{\delta p_{A}}= & M\left(\theta^{B} *\left(p_{B} \wedge \theta^{A}\right)-\frac{1}{2} \theta^{A} *\left(p_{B} \wedge \theta^{B}\right)\right), \\
\frac{\delta S_{2}}{\delta \theta^{A}}= & M\left(\frac{1}{2} \epsilon^{D}{ }_{B C A} \theta^{B} \wedge \theta^{C} * d p_{D}-\left(*^{B}\right) *_{A}^{\prime} d p_{B}\right), \\
\frac{\delta S_{2}}{\delta p_{A}}= & d\left(M \xi^{A}\right)=\xi^{A} d M+M d \xi^{A}, \\
\frac{\delta S_{3}}{\delta \theta^{A}}= & d\left(M \theta^{B} *\left(d \theta_{B} \wedge \theta_{A}\right)-\frac{M^{A}}{2} \theta_{A} *\left(d \theta_{B} \wedge \theta^{B}\right)\right)+M\left(d \theta_{B} *\left(d \theta_{A} \wedge \theta^{B}\right)\right. \\
& -\frac{1}{2} d \theta_{A} *\left(d \theta_{B} \wedge \theta^{B}\right)+\frac{1}{2}\left(d \theta_{C} \wedge \theta^{B}\right) *_{A}^{\prime}\left(d \theta_{B} \wedge \theta^{C}\right) \\
& \left.-\frac{1}{4}\left(d \theta_{B} \wedge \theta^{B}\right) *_{A}^{\prime}\left(d \theta_{C} \wedge \theta^{C}\right)\right),
\end{aligned}
$$




$$
\frac{\delta S_{3}}{\delta p_{A}}=0
$$

Let us begin the calculations with the bracket $\left\{S_{1}(M), S_{1}\left(M^{\prime}\right)\right\}$ :

$$
\left\{S_{1}(M), S_{1}\left(M^{\prime}\right)\right\}=\int_{\Sigma}\left(\frac{\delta S_{1}(M)}{\delta \theta^{A}} \wedge \frac{\delta S_{1}\left(M^{\prime}\right)}{\delta p_{A}}-\left(M \leftrightarrow M^{\prime}\right)\right)=0
$$

because the first term under the integral is symmetric in $M$ and $M^{\prime}$. It was shown in [9] that

$$
\left.\left\{S_{2}(M), S_{2}\left(M^{\prime}\right)\right\}=-\int_{\Sigma}(\vec{m}\lrcorner \theta^{A}\right) \wedge d p_{A},
$$

where

$$
m:=M d M^{\prime}-M^{\prime} d M
$$

Because $S_{3}(M)$ does not depend on the momenta $\left\{S_{3}(M), S_{3}\left(M^{\prime}\right)\right\}=0$. Next,

$$
\begin{aligned}
\left\{S_{1}(M), S_{2}\left(M^{\prime}\right)\right\}-\left(M \leftrightarrow M^{\prime}\right)= & \int_{\Sigma} m \wedge p_{B} *\left(\xi^{A} p_{A} \wedge \theta^{B}\right) \\
& -\frac{1}{2} m \wedge \xi^{A} p_{A} *\left(p_{B} \wedge \theta^{B}\right)
\end{aligned}
$$

— to obtain the result we removed terms symmetric in $M$ and $M^{\prime}$ and applied (3.3).

$$
\begin{aligned}
\left\{S_{2}(M), S_{3}\left(M^{\prime}\right)\right\}-\left(M \leftrightarrow M^{\prime}\right)= & \int_{\Sigma} m \wedge d \theta_{B} *\left(\xi^{A} d \theta_{A} \wedge \theta^{B}\right) \\
& -\frac{1}{2} m \wedge \xi^{A} d \theta_{A} *\left(d \theta_{B} \wedge \theta^{B}\right)
\end{aligned}
$$

- here there vanished terms symmetric in $M$ and $M^{\prime}$ and one being an exact threeform (recall that $\Sigma$ is a compact manifold without boundary); two terms vanished due to $(3.3)$.

$$
\begin{aligned}
& \left\{S_{3}(M), S_{1}\left(M^{\prime}\right)\right\}-\left(M \leftrightarrow M^{\prime}\right) \\
& =\int_{\Sigma}-m \wedge\left(\theta^{B} \wedge \theta^{C} *\left(d \theta_{B} \wedge \theta_{A} *\left(p_{C} \wedge \theta^{A}\right)\right)\right. \\
& \left.\quad-\frac{1}{2} \theta_{A} *\left(p_{C} \wedge \theta^{A}\right) \wedge \theta^{C} *\left(d \theta_{B} \wedge \theta^{B}\right)-\frac{1}{2} \theta^{B} \wedge \theta^{A} *\left(d \theta_{B} \wedge \theta_{A}\right) *\left(p_{C} \wedge \theta^{C}\right)\right)
\end{aligned}
$$




$$
\begin{aligned}
= & \int_{\Sigma}-m \wedge \theta^{B} \wedge \theta^{A} *\left(d \theta_{B} \wedge * p_{A}\right)-\frac{1}{2} m \wedge \theta^{A} \wedge * p_{A} *\left(d \theta_{B} \wedge \theta^{B}\right) \\
& +\frac{1}{2} m \wedge \theta^{B} \wedge * d \theta_{B} *\left(p_{A} \wedge \theta^{A}\right),
\end{aligned}
$$

where in the first step some terms disappeared by virtue of their symmetricity in $M$ and $M^{\prime}$; moreover, we used (3.16) in the last step.

Adding (3.28), (3.29), (3.30) and (3.31) we obtain

$$
\begin{aligned}
\left\{S(M), S\left(M^{\prime}\right)\right\}= & \left.\int_{\Sigma}-(\vec{m}\lrcorner \theta^{A}\right) \wedge d p_{A}+m \wedge p_{B} *\left(\xi^{A} p_{A} \wedge \theta^{B}\right) \\
& -\frac{1}{2} m \wedge \xi^{A} p_{A} *\left(p_{B} \wedge \theta^{B}\right)+m \wedge d \theta_{B} *\left(\xi^{A} d \theta_{A} \wedge \theta^{B}\right) \\
& -\frac{1}{2} m \wedge \xi^{A} d \theta_{A} *\left(d \theta_{B} \wedge \theta^{B}\right)-m \wedge \theta^{B} \wedge \theta^{A} *\left(d \theta_{B} \wedge * p_{A}\right) \\
& -\frac{1}{2} m \wedge \theta^{A} \wedge * p_{A} *\left(d \theta_{B} \wedge \theta^{B}\right)+\frac{1}{2} m \wedge \theta^{B} \wedge * d \theta_{B} *\left(p_{A} \wedge \theta^{A}\right) .
\end{aligned}
$$

\subsubsection{Isolating constraints}

Our goal now is to isolate constraints at the r.h.s. of (3.32), that is, to show that the r.h.s. of (3.32) is a sum of the constraints (2.5)-(2.8) smeared with some fields.

It is clear (see (2.8)) that the first term at the r.h.s. of (3.32)

$$
\left.\left.\int_{\Sigma}-(\vec{m}\lrcorner \theta^{A}\right) \wedge d p_{A}=V(\vec{m})+\int_{\Sigma} d \theta^{A} \wedge \vec{m}\right\lrcorner p_{A} .
$$

The second and the third terms of (3.32) are equal to

$$
\begin{aligned}
& B\left(\theta^{B} *\left(m \wedge p_{B}\right)-\frac{1}{2} m *\left(p_{B} \wedge \theta^{B}\right)\right) \\
& \quad+\int_{\Sigma}-*\left(m \wedge p_{B}\right) \theta^{B} \wedge \theta^{A} \wedge * d \theta_{A}+\frac{1}{2} *\left(p_{B} \wedge \theta^{B}\right) m \wedge \theta^{A} \wedge * d \theta_{A} .
\end{aligned}
$$

Similarly, the fourth and the fifth ones are equal to

$$
\begin{aligned}
& -R\left(\theta^{B} *\left(m \wedge d \theta_{B}\right)-\frac{1}{2} m *\left(d \theta_{B} \wedge \theta^{B}\right)\right) \\
& +\int_{\Sigma} *\left(m \wedge d \theta_{B}\right) \theta^{B} \wedge \theta^{A} \wedge * p_{A}-\frac{1}{2} *\left(d \theta_{B} \wedge \theta^{B}\right) m \wedge \theta^{A} \wedge * p_{A} .
\end{aligned}
$$


Thus (3.32) can be rewritten as follows:

$$
\begin{aligned}
\left\{S(M), S\left(M^{\prime}\right)\right\}= & V(\vec{m})+B\left(\theta^{B} *\left(m \wedge p_{B}\right)-\frac{1}{2} m *\left(p_{B} \wedge \theta^{B}\right)\right) \\
& -R\left(\theta^{B} *\left(m \wedge d \theta_{B}\right)-\frac{1}{2} m *\left(d \theta_{B} \wedge \theta^{B}\right)\right)+\text { remaining terms }
\end{aligned}
$$

where the remaining terms read

$$
\begin{aligned}
& \int_{\Sigma}-*\left(m \wedge p_{B}\right) \theta^{B} \wedge \theta^{A} \wedge * d \theta_{A}+*\left(m \wedge d \theta_{B}\right) \theta^{B} \wedge \theta^{A} \wedge * p_{A} \\
& \quad+*\left(p_{B} \wedge \theta^{B}\right) m \wedge \theta^{A} \wedge * d \theta_{A}-*\left(d \theta_{B} \wedge \theta^{B}\right) m \wedge \theta^{A} \wedge * p_{A} \\
& \left.\quad-m \wedge \theta^{B} \wedge \theta^{A} *\left(d \theta_{B} \wedge * p_{A}\right)+d \theta^{A} \wedge \vec{m}\right\lrcorner p_{A} .
\end{aligned}
$$

Now we will transform the remaining terms (3.34) to a form which will be a convenient starting point for isolating constraints. The first term in (3.34)

$$
\begin{aligned}
& -*\left(m \wedge p_{B}\right) \theta^{B} \wedge \theta^{A} \wedge * d \theta_{A}=-m \wedge p_{B} *\left(\theta^{B} \wedge \theta^{A} \wedge * d \theta_{A}\right) \\
& \left.=-\left(\vec{\theta}^{B}\right\lrcorner m\right) \theta^{A} \wedge * d \theta_{A} \wedge * p_{B}+m \wedge *\left(\theta^{A} \wedge * d \theta_{A}\right) \wedge *\left(\theta^{B} \wedge * p_{B}\right),
\end{aligned}
$$

where we used in turn (3.6) and (3.5). Similarly, the second term in (3.34)

$$
\begin{aligned}
& *\left(m \wedge d \theta_{B}\right) \theta^{B} \wedge \theta^{A} \wedge * p_{A} \\
& \left.\quad=\left(\vec{\theta}^{B}\right\lrcorner m\right) \wedge \theta^{A} \wedge * p_{A} \wedge * d \theta_{B}-m \wedge *\left(\theta^{A} \wedge * p_{A}\right) \wedge *\left(\theta^{B} \wedge * d \theta_{B}\right) .
\end{aligned}
$$

Next, we transform the third term in (3.34):

$$
\begin{aligned}
m & \left.\wedge \theta^{A} \wedge * d \theta_{A} *\left(p_{B} \wedge \theta^{B}\right)=\vec{\theta}^{B}\right\lrcorner\left(m \wedge \theta^{A} \wedge * d \theta_{A}\right) \wedge * p_{B} \\
= & \left.\left(\vec{\theta}^{B}\right\lrcorner m\right) \wedge \theta^{A} \wedge * d \theta_{A} \wedge * p_{B}-m \wedge * d \theta^{A} \wedge * p_{A}-m \wedge *\left(\xi^{A} d \theta_{A}\right) \wedge *\left(\xi^{B} p_{B}\right) \\
& +m \wedge \theta^{A} \wedge * p_{B} *\left(d \theta_{A} \wedge \theta^{B}\right),
\end{aligned}
$$

where in the first step we used (3.6), then (3.7) and (3.6) again. Similarly, the fourth term in (3.34)

$$
\begin{aligned}
-m \wedge \theta^{A} \wedge * p_{A} *\left(d \theta_{B} \wedge \theta^{B}\right)= & \left.-\left(\vec{\theta}^{B}\right\lrcorner m\right) \theta^{A} \wedge * p_{A} \wedge * d \theta_{B} \\
& \left.+m \wedge\left(\vec{\theta}^{B}\right\lrcorner \theta^{A}\right) * p_{A} \wedge * d \theta_{B} \\
& -m \wedge \theta^{A} \wedge * d \theta_{B} *\left(p_{A} \wedge \theta^{B}\right) .
\end{aligned}
$$

By virtue of (3.5) the last term in (3.34) can be expresses as follows [9]:

$$
\left.d \theta^{A} \wedge \vec{m}\right\lrcorner p_{A}=d \theta^{A} \wedge *\left(* p_{A} \wedge m\right)=* p_{A} \wedge m \wedge * d \theta^{A}=m \wedge * d \theta^{A} \wedge * p_{A} .
$$

Now it is easy to see that the following pairs 
1. the first term at the r.h.s. of (3.35) and the first term at the r.h.s. of (3.37),

2. the first term at the r.h.s. of (3.36) and the first term at the r.h.s. of (3.38),

3. the second term at the r.h.s. of (3.37) and (3.39)

sum up to zero. Moreover, the second term at the r.h.s. of (3.35) is equal to the second term at the r.h.s. of (3.36). Consequently, the terms (3.34) can be expresses as

$$
\begin{aligned}
& \left.\int_{\Sigma}\left(\vec{\theta}^{B}\right\lrcorner \theta^{A}\right) m \wedge * p_{A} \wedge * d \theta_{B}-m \wedge \theta^{A} \wedge * d \theta_{B} *\left(p_{A} \wedge \theta^{B}\right) \\
& \quad+m \wedge \theta^{A} \wedge * p_{B} *\left(d \theta_{A} \wedge \theta^{B}\right)+m \wedge *\left(\xi^{A} p_{A}\right) \wedge *\left(\xi^{B} d \theta_{B}\right) \\
& \quad-m \wedge \theta^{B} \wedge \theta^{A} *\left(d \theta_{B} \wedge * p_{A}\right)+2 m \wedge *\left(\theta^{A} \wedge * d \theta_{A}\right) \wedge *\left(\theta^{B} \wedge * p_{B}\right) .
\end{aligned}
$$

Note now that the term above containing $\xi^{A}$ can be transformed as follows:

$$
\begin{aligned}
\int_{\Sigma} & m \wedge *\left(\xi^{A} p_{A}\right) \wedge *\left(\xi^{B} d \theta_{B}\right) \\
= & \int_{\Sigma}-*\left[m \wedge *\left(\xi^{B} d \theta_{B}\right)\right] \wedge\left(\xi^{A} p_{A}\right)=-B\left(*\left(m \wedge \xi^{B} * d \theta_{B}\right)\right) \\
& +\int_{\Sigma} *\left[m \wedge *\left(\xi^{B} d \theta_{B}\right)\right] \wedge \theta^{A} \wedge * d \theta_{A}=-B\left(*\left(m \wedge \xi^{B} * d \theta_{B}\right)\right) \\
& -\int_{\Sigma} *\left[m \wedge *\left(\theta^{A} \wedge * d \theta_{A}\right)\right] \wedge \xi^{B} d \theta_{B}=-B\left(*\left(m \wedge \xi^{B} * d \theta_{B}\right)\right) \\
& +R\left(*\left[m \wedge *\left(\theta^{A} \wedge * d \theta_{A}\right)\right]\right)-\int_{\Sigma} m \wedge *\left(\theta^{A} \wedge * d \theta_{A}\right) \wedge *\left(\theta^{B} \wedge * p_{B}\right) .
\end{aligned}
$$

Gathering the result above, (3.40) and (3.33) we obtain

$$
\begin{aligned}
& \left\{S(M), S\left(M^{\prime}\right)\right\} \\
& =V(\vec{m})+B\left(\theta^{B} *\left(m \wedge p_{B}\right)-\frac{1}{2} m *\left(p_{B} \wedge \theta^{B}\right)\right) \\
& \quad-R\left(\theta^{B} *\left(m \wedge d \theta_{B}\right)-\frac{1}{2} m *\left(d \theta_{B} \wedge \theta^{B}\right)\right) \\
& \quad-B\left(*\left(m \wedge \xi^{B} * d \theta_{B}\right)\right)+R\left(*\left[m \wedge *\left(\theta^{A} \wedge * d \theta_{A}\right)\right]\right)+\text { remaining terms, }
\end{aligned}
$$


where the remaining terms read now

$$
\begin{gathered}
\left.\int_{\Sigma}\left(\vec{\theta}^{B}\right\lrcorner \theta^{A}\right) m \wedge * p_{A} \wedge * d \theta_{B}-m \wedge \theta^{A} \wedge * d \theta_{B} *\left(p_{A} \wedge \theta^{B}\right)+m \wedge \theta^{A} \wedge * p_{B} *\left(d \theta_{A} \wedge \theta^{B}\right) \\
-m \wedge \theta^{B} \wedge \theta^{A} *\left(d \theta_{B} \wedge * p_{A}\right)+m \wedge *\left(\theta^{A} \wedge * d \theta_{A}\right) \wedge *\left(\theta^{B} \wedge * p_{B}\right)
\end{gathered}
$$

Now let us show that the remaining terms (3.43) can be expressed as $R(b)$ with some one-form $b$. By shifting the contraction $\left.\vec{\theta}^{B}\right\lrcorner$ in the first term above and using (3.6) one can easily show that the sum of the first and the second terms in (3.43) reads

$$
\begin{aligned}
\left.\int_{\Sigma} \vec{\theta}^{B}\right\lrcorner\left(m \wedge * d \theta_{B}\right) \wedge \theta^{A} \wedge * p_{A}= & \left.R\left(\vec{\theta}^{B}\right\lrcorner\left(m \wedge * d \theta_{B}\right)\right) \\
& \left.+\int_{\Sigma} \vec{\theta}^{B}\right\lrcorner\left(m \wedge * d \theta_{B}\right) \wedge \xi^{A} d \theta_{A} .
\end{aligned}
$$

Expressing the third term in (3.43) by means of the components of the variables and the covariant derivative $\nabla_{a}$ and using (3.10) and (3.12) we obtain

$$
\begin{aligned}
m \wedge \theta^{A} \wedge * p_{B} *\left(d \theta_{A} \wedge \theta^{B}\right)= & m \wedge \theta_{i}^{A} p_{B}{ }^{a b} d x^{i} \wedge d x^{c}\left(\nabla_{a} \theta_{A b}\right) \theta_{c}^{B} \\
& +m \wedge \theta_{i}^{A} p_{B}{ }^{b c} d x^{i} \wedge d x^{a}\left(\nabla_{a} \theta_{A b}\right) \theta_{c}^{B} \\
& +m \wedge \theta_{i}^{A} p_{B}{ }^{c a} d x^{i} \wedge d x^{b}\left(\nabla_{a} \theta_{A b}\right) \theta_{c}^{B}
\end{aligned}
$$

Similarly, the fourth term in (3.43)

$$
-m \wedge \theta^{B} \wedge \theta^{A} *\left(d \theta_{B} \wedge * p_{A}\right)=-m \wedge \theta_{i}^{B} \theta_{c}^{A} d x^{i} \wedge d x^{c}\left(\nabla_{a} \theta_{B b}\right) p_{A}^{a b} .
$$

Consequently the sum of the third and the fourth terms in (3.43) is of the following form:

$$
\begin{aligned}
m & \wedge \theta_{i}^{A} p_{B}^{b c} d x^{i} \wedge d x^{a}\left(\nabla_{a} \theta_{A b}\right) \theta_{c}^{B}+m \wedge \theta_{i}^{A} p_{B}{ }^{c a} d x^{i} \wedge d x^{b}\left(\nabla_{a} \theta_{A b}\right) \theta_{c}^{B} \\
& \left.=-m \wedge\left(\theta^{B c} p_{B c}{ }^{b}\right)\left(d \theta_{A}\right)_{b a} d x^{a} \wedge \theta^{A}=-m \wedge\left[\overline{\left.\left(\vec{\theta}^{B}\right\lrcorner p_{B}\right)}\right\lrcorner d \theta^{A}\right] \wedge \theta^{A} \\
& =-m \wedge *\left[* d \theta_{A} \wedge *\left(* p_{B} \wedge \theta^{B}\right)\right] \wedge \theta^{A}=-*\left[*\left(m \wedge \theta^{A}\right) \wedge * d \theta_{A}\right] \wedge \theta^{B} \wedge * p_{B}
\end{aligned}
$$

-here in the fourth step we used (3.5). Thus the sum of the third and the fourth terms in (3.43) once integrated over $\Sigma$ reads:

$$
R\left(-*\left[*\left(m \wedge \theta^{B}\right) \wedge * d \theta_{B}\right]\right)+\int_{\Sigma}-*\left[*\left(m \wedge \theta^{B}\right) \wedge * d \theta_{B}\right] \wedge \xi^{A} d \theta_{A}
$$


Finally, the last term in (3.43) can be easily transformed to

$$
R\left(*\left[m \wedge *\left(\theta^{B} \wedge * d \theta_{B}\right)\right]\right)+\int_{\Sigma} *\left[m \wedge *\left(\theta^{B} \wedge * d \theta_{B}\right)\right] \wedge \xi^{A} d \theta_{A}
$$

Gathering the three results (3.44), (3.45) and (3.46) we conclude that the terms (3.43) can be expressed as

$$
\begin{aligned}
& \left.R\left(\vec{\theta}^{B}\right\lrcorner\left(m \wedge * d \theta_{B}\right)-*\left[*\left(m \wedge \theta^{B}\right) \wedge * d \theta_{B}\right]+*\left[m \wedge *\left(\theta^{B} \wedge * d \theta_{B}\right)\right]\right) \\
& \quad+\text { terms independent of } p_{A},
\end{aligned}
$$

where the terms independent of $p_{A}$ read

$$
\begin{aligned}
\int_{\Sigma} & \left.\vec{\theta}^{B}\right\lrcorner\left(m \wedge * d \theta_{B}\right) \wedge \xi^{A} d \theta_{A}-*\left[*\left(m \wedge \theta^{B}\right) \wedge * d \theta_{B}\right] \wedge \xi^{A} d \theta_{A}+*\left[m \wedge *\left(\theta^{B} \wedge * d \theta_{B}\right)\right] \wedge \xi^{A} d \theta_{A} \\
\quad & \left.\left.\left.\int_{\Sigma} m \xi^{A} \wedge\left[-* d \theta_{B} \wedge \vec{\theta}^{B}\right\lrcorner d \theta_{A}+*\left(\vec{\theta}^{B}\right\lrcorner\left(* d \theta_{B} \wedge * d \theta_{A}\right)\right)-\vec{\theta}^{B}\right\lrcorner d \theta_{B} \wedge * d \theta_{A}\right],
\end{aligned}
$$

where we isolated the factor $m \xi^{A}$ by means of (3.6) and (3.5). It is not difficult to show that the terms in the square brackets in (3.48) sum up to zero. Consequently, the terms (3.43) are equal to

$$
\left.R\left(\vec{\theta}^{B}\right\lrcorner\left(m \wedge * d \theta_{B}\right)-*\left[*\left(m \wedge \theta^{B}\right) \wedge * d \theta_{B}\right]+*\left[m \wedge *\left(\theta^{B} \wedge * d \theta_{B}\right)\right]\right) .
$$

Setting this result to (3.42) we obtain

$$
\begin{aligned}
& \left\{S(M), S\left(M^{\prime}\right)\right\}=V(\vec{m})+B\left(\theta^{B} *\left(m \wedge p_{B}\right)-\frac{1}{2} m *\left(p_{B} \wedge \theta^{B}\right)\right) \\
& \quad-R\left(\theta^{B} *\left(m \wedge d \theta_{B}\right)-\frac{1}{2} m *\left(d \theta_{B} \wedge \theta^{B}\right)\right) \\
& \quad-B\left(*\left(m \wedge \xi^{B} * d \theta_{B}\right)\right)+R\left(*\left[m \wedge *\left(\theta^{A} \wedge * d \theta_{A}\right)\right]\right) \\
& \left.\quad+R\left(\vec{\theta}^{B}\right\lrcorner\left(m \wedge * d \theta_{B}\right)-*\left[*\left(m \wedge \theta^{B}\right) \wedge * d \theta_{B}\right]+*\left[m \wedge *\left(\theta^{B} \wedge * d \theta_{B}\right)\right]\right) .
\end{aligned}
$$

\subsubsection{Another form of $\left\{S(M), S\left(M^{\prime}\right)\right\}$}

Let us now transform (3.50) to a form in which both constraints $B$ given by (2.5) and $R$ defined by (2.6) appear on an equal footing. Consider the following transformation:

$$
p_{A} \mapsto-d \theta_{A}, \quad d \theta_{A} \mapsto p_{A}
$$


It is easy to see that under this transformation

$$
B\left(a\left(p_{A}, d \theta_{B}\right)\right) \mapsto R\left(a\left(-d \theta_{A}, p_{B}\right)\right), \quad R\left(b\left(p_{A}, d \theta_{B}\right)\right) \mapsto-B\left(b\left(-d \theta_{A}, p_{B}\right)\right)
$$

Assume now that a function $F$ on the phase space is mapped by the transformations (3.51) to a function $G$ and that we know from somewhere else that $F=G$. Then $F=(F+G) / 2$. We will use this fact to transform (3.50) to the desired form.

Let us now express the formula (3.41) in the following form:

$$
\begin{aligned}
& \int_{\Sigma} m \wedge *\left(\xi^{A} p_{A}\right) \wedge *\left(\xi^{B} d \theta_{B}\right)+m \wedge *\left(\theta^{A} \wedge * d \theta_{A}\right) \wedge *\left(\theta^{B} \wedge * p_{B}\right) \\
& =-B\left(*\left(m \wedge \xi^{B} * d \theta_{B}\right)\right)+R\left(*\left[m \wedge *\left(\theta^{A} \wedge * d \theta_{A}\right)\right]\right)
\end{aligned}
$$

Note now that the 1.h.s. of the identity above is invariant with respect to the transformation (3.51). Consequently, the r.h.s. has to be invariant too and treating the r.h.s. as a function $F$ by virtue of (3.52) we obtain from it a function $G$ such that $F=G$. Thus the r.h.s. is equal to $(F+G) / 2$ and reads

$$
\begin{aligned}
& \frac{1}{2} R\left(-*\left(m \wedge \xi^{B} * p_{B}\right)+*\left[m \wedge *\left(\theta^{A} \wedge * d \theta_{A}\right)\right]\right) \\
& \quad-\frac{1}{2} B\left(*\left(m \wedge \xi^{B} * d \theta_{B}\right)+*\left[m \wedge *\left(\theta^{A} \wedge * p_{A}\right)\right]\right) .
\end{aligned}
$$

In this way we obtained an alternative expression for the fourth and the fifth terms at the r.h.s. of (3.50).

Similarly, the expression (3.43) is also invariant with respect to (3.51). On the other hand, (3.43) is equal to the last term at the r.h.s. of (3.50) (see the sentence containing (3.49)) which means that the term can be treated as a function $F$ and expressed as

$$
\begin{aligned}
& \left.\frac{1}{2} R\left(\vec{\theta}^{B}\right\lrcorner\left(m \wedge * d \theta_{B}\right)-*\left[*\left(m \wedge \theta^{B}\right) \wedge * d \theta_{B}\right]+*\left[m \wedge *\left(\theta^{B} \wedge * d \theta_{B}\right)\right]\right) \\
& \left.\quad-\frac{1}{2} B\left(\vec{\theta}^{B}\right\lrcorner\left(m \wedge * p_{B}\right)-*\left[*\left(m \wedge \theta^{B}\right) \wedge * p_{B}\right]+*\left[m \wedge *\left(\theta^{B} \wedge * p_{B}\right)\right]\right) .
\end{aligned}
$$

Setting the results (3.53) and (3.54) to (3.50) after some simple calculations with application of (3.5) and (3.6) we obtain the final form $\left\{S(M), S\left(M^{\prime}\right)\right\}$ :

$$
\begin{aligned}
& \left\{S(M), S\left(M^{\prime}\right)\right\}=V(\vec{m})+B\left(\theta^{B} *\left(m \wedge p_{B}\right)-\frac{1}{2} *\left(m \wedge \xi^{B} * d \theta_{B}\right)\right. \\
& \left.\quad-*\left[m \wedge *\left(\theta^{B} \wedge * p_{B}\right)\right]-\frac{1}{2} *\left(* m \wedge \theta^{B}\right) * p_{B}+\frac{1}{2} *\left[*\left(m \wedge \theta^{B}\right) \wedge * p_{B}\right]\right) \\
& \quad+R\left(-\theta^{B} *\left(m \wedge d \theta_{B}\right)-\frac{1}{2} *\left(m \wedge \xi^{B} * p_{B}\right)\right.
\end{aligned}
$$




$$
\left.+*\left[m \wedge *\left(\theta^{B} \wedge * d \theta_{B}\right)\right]+\frac{1}{2} *\left(* m \wedge \theta^{B}\right) * d \theta_{B}-\frac{1}{2} *\left[*\left(m \wedge \theta^{B}\right) \wedge * d \theta_{B}\right]\right)
$$

Note that the sum of the constraints $B$ and $R$ at the r.h.s. of (3.55) is explicitely invariant with respect to the transformation (3.51).

\subsection{Poisson bracket of $R(b)$ and $S(M)$}

Recall that the constraints $R(b)$ and $S(M)$ are defined by, respectively, (2.6) and (2.7). To show that the bracket $\{R(b), S(M)\}$ is a sum of the constraints (2.5)-(2.8) smeared with some fields we will proceed according to the following prescription. The bracket under consideration can be expressed as

$$
\{R(b), S(M)\}=\sum_{i=1}^{2} \sum_{j=1}^{3}(-1)^{i+1}\left\{R_{i}(b), S_{j}(M)\right\},
$$

where the functionals at the r.h.s. are given by (3.21) and (3.26). It is not difficult to see that each bracket in the sum is either quadratic in $p_{A}$, linear in $p_{A}$ or independent of $p_{A}$. So we will first calculate the brackets and then we will gather similar terms according to the classification. Next, it will turn out that the terms quadratic in $p_{A}$ can be re-expressed as a constraint plus a term linear in $p_{A}$. Then it will turn out that all the linear terms can be re-expressed as some constraints plus terms independent of $p_{A}$ and that all the terms independent of $p_{A}$ sum up to zero.

Except the prescription we will need some formulae and identities which will make easier the calculations.

\subsubsection{Auxiliary formulae}

The following formulae will be used in the sequel while calculating $\{R(b), S(M)\}$ (they are also very useful in calculating $\{B(a), S(M)\}$ ):

$$
\begin{aligned}
\left.\vec{\theta}_{A}\right\lrcorner * \xi^{B}= & -\frac{1}{2} \epsilon^{B}{ }_{C D A} \theta^{C} \wedge \theta^{D}+\xi_{A} * \theta^{B}, \\
-\frac{1}{2} \epsilon_{B C D}^{A} \theta^{B} \wedge \theta^{C} \wedge \alpha= & \left.-\xi_{D} \alpha \wedge * \theta^{A}+\left(* \xi^{A}\right) \vec{\theta}_{D}\right\lrcorner \alpha \\
\left(\alpha *_{A}^{\prime} \beta\right) \wedge \gamma= & \left.\left.\alpha \wedge * \beta \vec{\theta}_{A}\right\lrcorner \gamma-\left[\left(\vec{\theta}_{A}\right\lrcorner \alpha\right) \wedge * \beta+(\alpha \leftrightarrow \beta)\right] \wedge \gamma \\
= & {\left.\left.\left[(-1)^{k} \alpha \wedge\left(\vec{\theta}_{A}\right\lrcorner * \beta\right)-\left(\vec{\theta}_{A}\right\lrcorner \beta\right) \wedge * \alpha\right] \wedge \gamma, } \\
\left(\alpha *_{A}^{\prime} \beta\right) \wedge \frac{\delta S_{1}(M)}{\delta p_{A}}= & M\left(k-\frac{5}{2}\right) \alpha \wedge * \beta *\left(p_{A} \wedge \theta^{A}\right) \\
& +(-1)^{4-k} M\left[*\left(\alpha \wedge \theta^{A}\right) \wedge * p_{A} \wedge \beta+(\alpha \leftrightarrow \beta)\right], \\
\alpha^{A} \wedge \beta_{A}= & \left.\left.\theta_{A} \wedge \vec{\theta}^{B}\right\lrcorner \alpha^{A} \wedge \beta_{B}+(-1)^{k} \theta_{A} \wedge \alpha^{A} \wedge \vec{\theta}^{B}\right\lrcorner \beta_{B} \\
& -\xi_{A} \alpha^{A} \wedge \xi^{B} \beta_{B}, \\
d\left(*\left(* \theta^{A} \wedge \theta^{B}\right)\right)= & \xi^{B} d \xi^{A}+\xi^{A} d \xi^{B},
\end{aligned}
$$


where in (3.57) $\alpha$ is a one-form, and in (3.58) $\alpha$ and $\beta$ are $k$-forms and $\gamma$ a one-form, in (3.59) $\alpha$ and $\beta$ are $k$-forms and finally in (3.60) $\alpha^{A}$ is a $k$-form, while $\beta_{A}$ is a $(3-k)$-form. Moreover, we will apply the following two identities:

$$
\begin{array}{r}
\alpha \wedge *(* \gamma \wedge \beta)-* \alpha *(\beta \wedge \gamma)-\beta \wedge *(* \gamma \wedge \alpha)+* \beta *(\alpha \wedge \gamma)=0 \\
{[\alpha \wedge *(\kappa \wedge \beta)]-\alpha *(\beta \wedge * \kappa)-*[\beta \wedge *(\kappa \wedge \alpha)]+\beta *(\alpha \wedge * \kappa)=0}
\end{array}
$$

where $\alpha, \beta$ and $\kappa$ are one-forms, and $\gamma$ is a two-form.

Note that if in (3.58) $\alpha$ and $\beta$ are three-forms then the formula can be simplified further. Indeed, in this case $* \alpha$ and $* \beta$ are zero-forms thus

$$
\left.\left.\left(\vec{\theta}_{A}\right\lrcorner \alpha\right) \wedge * \beta \wedge \gamma=\alpha \wedge * \beta \vec{\theta}_{A}\right\lrcorner \gamma
$$

and setting this equality to the r.h.s. of the first line of (3.58) we obtain

$$
\left.\left(\alpha *_{A}^{\prime} \beta\right) \wedge \gamma=-\alpha \wedge * \beta \vec{\theta}_{A}\right\lrcorner \gamma .
$$

Using this result we can also simplify (3.59) —if $\alpha$ and $\beta$ are three-forms then setting to $(3.64) \gamma=\left(\delta S_{1}(M)\right) /\left(\delta p_{A}\right)$ we obtain

$$
\left(\alpha *_{A}^{\prime} \beta\right) \wedge \frac{\delta S_{1}(M)}{\delta p_{A}}=\frac{M}{2} \alpha \wedge * \beta *\left(p_{A} \wedge \theta^{A}\right) .
$$

Proof of (3.56) Recall that the functions $\xi^{B}$ are given by the formula (2.2). Using it we obtain

$$
\begin{aligned}
\left.\vec{\theta}_{A}\right\lrcorner * \xi^{B} & \left.=-\frac{1}{2} \epsilon^{B}{ }_{C D E}\left(\vec{\theta}_{A}\right\lrcorner \theta^{C}\right) \theta^{D} \wedge \theta^{E} \\
& =-\frac{1}{2} \epsilon^{B}{ }_{A D E} \theta^{D} \wedge \theta^{E}-\frac{1}{2} \epsilon^{B}{ }_{C D E} \xi_{A} \xi^{C} \theta^{D} \wedge \theta^{E} \\
& =-\frac{1}{2} \epsilon^{B}{ }_{C D A} \theta^{C} \wedge \theta^{D}+\xi_{A} * \theta^{B},
\end{aligned}
$$

where in the second step we used (3.7), and in the last one (3.18).

Proof of (3.57) By virtue of (3.7) and (3.18) the 1.h.s. of (3.57) can be transformed as follows:

$$
\begin{aligned}
-\frac{1}{2} \epsilon_{B C D}^{A} \theta^{B} \wedge \theta^{C} \wedge \alpha & \left.=-\frac{1}{2} \epsilon^{A}{ }_{B C E} \theta^{B} \wedge \theta^{C} \wedge\left(\vec{\theta}_{D}\right\lrcorner \theta^{E}-\xi_{D} \xi^{E}\right) \alpha \\
& \left.=-\frac{1}{2} \epsilon^{A}{ }_{B C E} \theta^{B} \wedge \theta^{C} \wedge\left(\vec{\theta}_{D}\right\lrcorner \theta^{E}\right) \alpha-\left(* \theta^{A}\right) \wedge \xi_{D} \alpha .
\end{aligned}
$$


Shifting the contraction $\left.\vec{\theta}_{D}\right\lrcorner$ in the first of the two resulting terms and applying once again (3.7) and (3.18) we obtain

$$
\begin{aligned}
& -\frac{1}{2} \epsilon_{B C D}^{A} \theta^{B} \wedge \theta^{C} \wedge \alpha=\epsilon^{A}{ }_{D C E} \theta^{C} \wedge \theta^{E} \wedge \alpha \\
& \left.-\frac{1}{2} \epsilon^{A}{ }_{B C E} \theta^{B} \wedge \theta^{C} \wedge \theta^{E}\left(\vec{\theta}_{D}\right\lrcorner \alpha\right)-3\left(* \theta^{A}\right) \wedge \xi_{D} \alpha .
\end{aligned}
$$

Now to justify (3.57) it is enough to note that (1) the first term on the r.h.s of the equation above is proportional to the term on the l.h.s. and (2) the second term on the r.h.s. by virtue of (2.2) is equal to $\left.3\left(*^{A}\right) \vec{\theta}_{D}\right\lrcorner \alpha$.

Proof of (3.58) Let us now consider the 1.h.s. of (3.58):

$$
\begin{aligned}
\left(\alpha *_{A}^{\prime} \beta\right) \wedge \gamma & \left.\left.\left.=\vec{\theta}^{B}\right\lrcorner\left(\eta_{A B} \alpha \wedge * \beta-\left[\left(\vec{\theta}_{A}\right\lrcorner \alpha\right) \wedge *\left(\vec{\theta}_{B}\right\lrcorner \beta\right)+(\alpha \leftrightarrow \beta)\right]\right) \wedge \gamma \\
& \left.\left.\left.\left.=\alpha \wedge * \beta\left(\vec{\theta}_{A}\right\lrcorner \gamma\right)-\left[\left(\vec{\theta}_{A}\right\lrcorner \alpha\right) \wedge *\left(\vec{\theta}_{B}\right\lrcorner \beta\right)+(\alpha \leftrightarrow \beta)\right] \vec{\theta}^{B}\right\lrcorner \gamma .
\end{aligned}
$$

By virtue of (3.5) and (3.8)

$$
\left.\left.\left.*\left(\vec{\theta}_{B}\right\lrcorner \beta\right) \vec{\theta}^{B}\right\lrcorner \gamma=* \beta \wedge \theta_{B} \wedge \vec{\theta}^{B}\right\lrcorner \gamma=* \beta \wedge \gamma .
$$

Setting this result to the r.h.s. of the previous equation we obtain the r.h.s. of the first line of (3.58). To obtain the result at the second line it is enough to shift the contraction $\left.\vec{\theta}_{A}\right\lrcorner$ in the term $\left.-\left(\vec{\theta}_{A}\right\lrcorner \alpha\right) \wedge * \beta \wedge \gamma$.

Proof of (3.59) By virtue of the first line of Eq. (3.58) just proven

$$
\begin{aligned}
\left(\alpha *_{A}^{\prime} \beta\right) \wedge \frac{\delta S_{1}(M)}{\delta p_{A}}= & \left.\left.\alpha \wedge * \beta \vec{\theta}_{A}\right\lrcorner \frac{\delta S_{1}(M)}{\delta p_{A}}-\left[\left(\vec{\theta}_{A}\right\lrcorner \alpha\right) \wedge * \beta+(\alpha \leftrightarrow \beta)\right] \wedge \frac{\delta S_{1}(M)}{\delta p_{A}} \\
= & \left.-\frac{M}{2} \alpha \wedge * \beta *\left(p_{A} \wedge \theta^{A}\right)-M\left[\left(\vec{\theta}_{A}\right\lrcorner \alpha\right) \wedge * \beta+(\alpha \leftrightarrow \beta)\right] \\
& \wedge\left(\theta^{B} *\left(p_{B} \wedge \theta^{A}\right)-\frac{1}{2} \theta^{A} *\left(p_{B} \wedge \theta^{B}\right)\right)
\end{aligned}
$$

Because $*\left(p_{B} \wedge \theta^{A}\right)$ is a zero-form (i.e. a function)

$$
\begin{aligned}
\left.\left(\vec{\theta}_{A}\right\lrcorner \alpha\right) *\left(p_{B} \wedge \theta^{A}\right) & =*\left(* \alpha \wedge \theta_{A}\right) *\left(p_{B} \wedge \theta^{A}\right) \\
& =*\left(* \alpha \wedge \theta_{A} \wedge *\left(p_{B} \wedge \theta^{A}\right)\right)=*\left(* \alpha \wedge * p_{B}\right),
\end{aligned}
$$

where the first equality holds true by virtue of (3.5) and the last one-due to (3.16). On the other hand due to (3.8)

$$
\left.\left.\left(\vec{\theta}_{A}\right\lrcorner \alpha\right) \wedge * \beta \wedge \theta^{A}=\theta^{A} \wedge\left(\vec{\theta}_{A}\right\lrcorner \alpha\right) \wedge * \beta=k \alpha \wedge * \beta .
$$


Setting the two results above to (3.66) we get

$$
\begin{aligned}
\left(\alpha *_{A}^{\prime} \beta\right) \wedge \frac{\delta S_{1}(M)}{\delta p_{A}}= & M\left(k-\frac{1}{2}\right) \alpha \wedge * \beta *\left(p_{A} \wedge \theta^{A}\right) \\
& -M\left[*\left(* \alpha \wedge * p_{A}\right) \wedge * \beta \wedge \theta^{A}+(\alpha \leftrightarrow \beta)\right] .
\end{aligned}
$$

The last step of the proof aims at simplifying the last term in the equation above:

$$
\begin{aligned}
*\left(* \alpha \wedge * p_{A}\right) \wedge * \beta \wedge \theta^{A} & =* \alpha \wedge * p_{A} \wedge *\left(* \beta \wedge \theta^{A}\right) \\
& \left.=* \alpha \wedge * p_{A} \wedge \vec{\theta}^{A}\right\lrcorner \beta \\
& \left.=(-1)^{3-k} \vec{\theta}^{A}\right\lrcorner\left(* \alpha \wedge * p_{A}\right) \wedge \beta \\
& =(-1)^{3-k} *\left(\alpha \wedge \theta^{A}\right) \wedge * p_{A} \wedge \beta+* \alpha \wedge \beta *\left(p_{A} \wedge \theta^{A}\right)
\end{aligned}
$$

(here we used (3.5) and (3.6)). Taking into account that $* \alpha \wedge \beta=\alpha \wedge * \beta$ we set the result above to (3.67) obtaining thereby (3.59).

Proof of (3.60) By virtue of (3.7)

$$
\left.\left.\alpha^{A} \wedge \beta_{A}=\left(\vec{\theta}^{B}\right\lrcorner \theta_{A}-\xi^{B} \xi_{A}\right) \alpha^{A} \wedge \beta_{B}=\vec{\theta}^{B}\right\lrcorner \theta_{A} \alpha^{A} \wedge \beta_{B}-\xi_{A} \alpha^{A} \wedge \xi^{B} \beta_{B} .
$$

Now to get (3.60) it is enough to shift the contraction $\left.\vec{\theta}^{B}\right\lrcorner$ in the first term at the r.h.s. of the equation above.

Proof of (3.61) First transform the 1.h.s. of (3.7) by means of (3.5) then act on the both sides of the resulting formula by $d$.

Proof of (3.62) Note that $\beta \wedge \gamma$ is a three form. Therefore

$$
* \alpha *(\beta \wedge \gamma)=*[*(\beta \wedge \gamma) \wedge \alpha]=\vec{\alpha}\lrcorner(\beta \wedge \gamma)=\vec{\alpha}\lrcorner \beta \wedge \gamma-\beta \wedge \vec{\alpha}\lrcorner \gamma
$$

where in the second step we used (3.5). Transforming similarly the term $* \beta *(\alpha \wedge \gamma)$ we obtain

$$
\begin{aligned}
& \alpha \wedge *(* \gamma \wedge \beta)-* \alpha *(\beta \wedge \gamma)-\beta \wedge *(* \gamma \wedge \alpha)+* \beta *(\alpha \wedge \gamma) \\
& \quad=\alpha \wedge \vec{\beta}\lrcorner \gamma-\vec{\alpha}\lrcorner \beta \wedge \gamma+\beta \wedge \vec{\alpha}\lrcorner \gamma-\beta \wedge \vec{\alpha}\lrcorner \gamma+\vec{\beta}\lrcorner \alpha \wedge \gamma-\alpha \wedge \vec{\beta}\lrcorner \gamma=0 .
\end{aligned}
$$

Proof of (3.63) Act by the Hodge operator $*$ on both sides of (3.62) and set $\kappa=* \gamma . \square$

Now we are ready to begin the calculations of $\{R(B), S(M)\}$. Let us recall that variations needed to calculate the bracket are given by (3.22) and (3.27). 


\subsubsection{Terms quadratic in $p_{A}$}

Terms quadratic in the momenta come form the Poisson bracket

$$
\begin{aligned}
\left\{R_{1}(b), S_{1}(M)\right\}= & \int_{\Sigma}\left[-b \wedge * p_{A}+\left(b \wedge \theta^{B}\right) *_{A}^{\prime} p_{B}\right] \wedge \frac{\delta S_{1}(M)}{\delta p_{A}} \\
& -M\left(p_{B} *\left(p_{A} \wedge \theta^{B}\right)-\frac{1}{2} p_{A} *\left(p_{B} \wedge \theta^{B}\right)\right) \wedge *\left(b \wedge \theta^{A}\right),
\end{aligned}
$$

where we used (3.15) to simplify the r.h.s. It is not difficult to see that $-b \wedge * p_{A} \wedge \frac{\delta S_{1}(M)}{\delta p_{A}}-M\left(p_{B} *\left(p_{A} \wedge \theta^{B}\right)-\frac{1}{2} p_{A} *\left(p_{B} \wedge \theta^{B}\right)\right) \wedge *\left(b \wedge \theta^{A}\right)=0$.

Let us now transform the remaining term in (3.68) — by virtue of (3.59)

$$
\begin{aligned}
& {\left[\left(b \wedge \theta^{B}\right) *_{A}^{\prime} p_{B}\right] \wedge \frac{\delta S_{1}(M)}{\delta p_{A}}=-\frac{M}{2} b \wedge \theta^{B} \wedge * p_{B} *\left(p_{A} \wedge \theta^{A}\right)} \\
& \quad+M *\left(b \wedge \theta^{B} \wedge \theta^{A}\right) \wedge * p_{A} \wedge p_{B}+M *\left(p_{B} \wedge \theta^{A}\right) \wedge * p_{A} \wedge b \wedge \theta^{B} .
\end{aligned}
$$

Note that $\left(b \wedge \theta^{B} \wedge \theta^{A}\right)$ is antisymmetric in $A$ and $B$ while $* p_{A} \wedge p_{B}$ is symmetric. Consequently, the second term at the r.h.s. above vanishes and

$$
\left\{R_{1}(b), S_{1}(M)\right\}=\int_{\Sigma}-\frac{M}{2} b \wedge \theta^{B} \wedge * p_{B} *\left(p_{A} \wedge \theta^{A}\right)+M *\left(p_{B} \wedge \theta^{A}\right) b \wedge \theta^{B} \wedge * p_{A} .
$$

\subsubsection{Terms linear in $p_{A}$}

Here we will calculate the brackets $\left\{R_{1}(b), S_{2}(M)\right\}$ and $\left\{R_{2}(b), S_{1}(M)\right\}$, which give terms linear in the momenta.

Considering $\left\{R_{1}(b), S_{2}(M)\right\}$ we immediately see that by virtue of (3.15) and (3.17)

$$
\frac{\delta S_{2}(M)}{\delta \theta^{A}} \wedge \frac{\delta R_{1}(b)}{\delta p_{A}}=0,
$$

thus

$$
\begin{aligned}
\left\{R_{1}(b), S_{2}(M)\right\}= & \int_{\Sigma}\left[-b \wedge * p_{A}+\left(b \wedge \theta^{B}\right) *_{A}^{\prime} p_{B}\right] \wedge d\left(M \xi^{A}\right)=\int_{\Sigma}-d M \wedge b \wedge *\left(\xi^{A} p_{A}\right) \\
& \left.-M b \wedge * p_{A} \wedge d \xi^{A}+M b \wedge \theta^{B} \wedge d \xi^{A} \vec{\theta}_{A}\right\lrcorner * p_{B} \\
& \left.-M \vec{\theta}_{A}\right\lrcorner p_{B} \wedge *\left(b \wedge \theta^{B}\right) \wedge d \xi^{A}
\end{aligned}
$$


where we used the second line of (3.58) and (3.3).

The other bracket,

$$
\begin{aligned}
\left\{R_{2}(b), S_{1}(M)\right\}= & \int_{\Sigma}\left[d\left(b \xi_{A}\right)+\left(b \wedge d \theta^{B}\right) *_{A}^{\prime}\left(* \xi_{B}\right)\right. \\
& \left.-\frac{1}{2} \epsilon_{D B C A} \theta^{B} \wedge \theta^{C} *\left(b \wedge d \theta^{D}\right)\right] \wedge \frac{\delta S_{1}(M)}{\delta p_{A}} .
\end{aligned}
$$

The last two terms in the square bracket above give together zero once multiplied by $\left(\delta S_{1}(M)\right) /\left(\delta p_{A}\right)$. Indeed, due to (3.65)

$$
\left[\left(b \wedge d \theta^{B}\right) *_{A}^{\prime}\left(* \xi_{B}\right)\right] \wedge \frac{\delta S_{1}(M)}{\delta p_{A}}=\frac{M}{2}\left(b \wedge \xi_{B} d \theta^{B}\right) *\left(p_{A} \wedge \theta^{A}\right)
$$

On the other hand,

$$
\begin{aligned}
-\frac{1}{2} \epsilon_{D B C A} \theta^{B} \wedge \theta^{C} \wedge \frac{\delta S_{1}(M)}{\delta p_{A}} *\left(b \wedge d \theta^{D}\right) & \left.=* \xi_{D} *\left(b \wedge d \theta^{D}\right) \vec{\theta}_{A}\right\lrcorner \frac{\delta S_{1}(M)}{\delta p_{A}} \\
& =-\frac{M}{2}\left(b \wedge \xi_{B} d \theta^{B}\right) *\left(p_{A} \wedge \theta^{A}\right)
\end{aligned}
$$

- the first equality holds by virtue of (3.57) and due to a fact that $\xi_{A}\left(\delta S_{1}(M)\right) /\left(\delta p_{A}\right)=$ $0\left(\left(\delta S_{1}(M)\right) /\left(\delta p_{A}\right)\right.$ is of the form $\theta_{i}^{A} \gamma^{i}{ }_{j} d x^{j}$ form some tensor field $\left.\gamma^{i}{ }_{j}\right)$, in the last step we used (3.7). The fact just mentioned and (3.6) allows us two express the only remaining term in (3.71) as follows:

$$
\left.d\left(b \xi_{A}\right) \wedge \frac{\delta S_{1}(M)}{\delta p_{A}}=M b \wedge \theta^{B} \wedge d \xi^{A} \vec{\theta}_{A}\right\lrcorner * p_{B}-\frac{M}{2} b \wedge \theta^{A} \wedge d \xi_{A} *\left(p_{B} \wedge \theta^{B}\right) .
$$

Gathering all the terms linear in $p_{A}$, that is, (3.70) and (3.72) we obtain

$$
\begin{aligned}
\left\{R_{1}(b), S_{2}(M)\right\}-\left\{R_{2}(b), S_{1}(M)\right\}= & \int_{\Sigma}-d M \wedge b \wedge *\left(\xi^{A} p_{A}\right)-M b \wedge * p_{A} \wedge d \xi^{A} \\
& \left.-M \vec{\theta}_{A}\right\lrcorner p_{B} \wedge *\left(b \wedge \theta^{B}\right) \wedge d \xi^{A} \\
& +\frac{M}{2} b \wedge \theta^{A} \wedge d \xi_{A} *\left(p_{B} \wedge \theta^{B}\right) .
\end{aligned}
$$

\subsubsection{Terms independent of $p_{A}$}

It turns out that the remaining three brackets, $\left\{R_{2}(b), S_{3}(M)\right\},\left\{R_{2}(b), S_{2}(M)\right\}$ and $\left\{R_{1}(b), S_{3}(M)\right\}$ do not depend on the momenta. The first bracket is zero since both 
$R_{2}(b)$ and $S_{3}(M)$ do not contain $p_{A}$. The second bracket contains a term $d\left(b \xi_{A}\right) \wedge$ $d\left(M \xi^{A}\right)$ being an exact three-form-the integral of this term over $\Sigma$ is zero hence

$$
\left\{R_{2}(b), S_{2}(M)\right\}=\int_{\Sigma}\left[\left(b \wedge d \theta^{B}\right) *_{A}^{\prime}\left(* \xi_{B}\right)-\frac{1}{2} \epsilon_{D B C A} \theta^{B} \wedge \theta^{C} *\left(b \wedge d \theta^{D}\right)\right] \wedge d\left(M \xi^{A}\right) .
$$

Applying (3.64), (3.18) and (3.57) it is not difficult to show that

$$
\left\{R_{2}(b), S_{2}(M)\right\}=\int_{\Sigma} d M \wedge * \theta_{D} *\left(b \wedge d \theta^{D}\right)
$$

Consider now the last bracket $\left\{R_{1}(b), S_{3}(M)\right\}$. Due to (3.15)

$$
\begin{aligned}
\left\{R_{1}(b), S_{3}(M)\right\}= & -\int_{\Sigma}\left(d\left[M \theta^{B} *\left(d \theta_{B} \wedge \theta_{A}\right)-\frac{M}{2} \theta_{A} *\left(d \theta_{B} \wedge \theta^{B}\right)\right]\right. \\
& \left.+M d \theta_{B} *\left(d \theta_{A} \wedge \theta^{B}\right)-\frac{M}{2} d \theta_{A} *\left(d \theta_{B} \wedge \theta^{B}\right)\right) \wedge *\left(b \wedge \theta^{A}\right) .
\end{aligned}
$$

Our goal now is to express the r.h.s. of the equation above as a sum of a term containing $M b$ and a one containing $d M$. To this end we first act by the operator $d$ on the factors constituting terms in the square brackets. Next, in those cases when it is possible, we use (3.16) to simplify $\theta_{A} \wedge *\left(b \wedge \theta^{A}\right)$ to $2 * b$ and $\theta_{A} \wedge *\left(d \theta_{B} \wedge \theta^{A}\right)$ to $* d \theta_{B}$, finally in all the terms containing $M$ and $* b$ we shift the Hodge operator $*$ to get $b$. Thus we obtain

$$
\begin{aligned}
\left\{R_{1}(b), S_{3}(M)\right\}= & -\int_{\Sigma} M b \wedge\left[-* d \theta^{A} \wedge * d \theta_{A}+\theta^{A} \wedge * d \theta^{B} *\left(d \theta_{A} \wedge \theta_{B}\right)\right. \\
& -\theta^{A} \wedge * d \theta_{A} *\left(d \theta_{B} \wedge \theta^{B}\right)-\theta^{A} \wedge *\left(\theta^{B} \wedge d *\left(d \theta_{B} \wedge \theta_{A}\right)\right) \\
& \left.-* d *\left(d \theta_{A} \wedge \theta^{A}\right)\right]+d M \wedge\left[\theta^{A} \wedge *\left(b \wedge * d \theta_{A}\right)\right. \\
& \left.-* b *\left(d \theta_{A} \wedge \theta^{A}\right)\right] .
\end{aligned}
$$

Note that since $* d \theta^{A}$ is a one-form the first term at the r.h.s. above vanishes. Thus the terms independent of $p_{A}$ read

$$
\begin{aligned}
& \left\{R_{1}(b), S_{3}(M)\right\}-\left\{R_{2}(b), S_{2}(M)\right\}=\int_{\Sigma} \text { the term containing } M b \\
& -\int_{\Sigma} d M \wedge\left[-* b *\left(\theta^{B} \wedge d \theta_{B}\right)-\theta^{B} \wedge *\left(* d \theta_{B} \wedge b\right)+* \theta^{B} *\left(b \wedge d \theta_{B}\right)\right] .
\end{aligned}
$$


Now by setting in (3.62) $\alpha=b, \beta=\theta^{B}$ and $\gamma=d \theta_{B}$ the last term above can be simplified to

$$
-\int_{\Sigma} d M \wedge\left[-b \wedge *\left(* d \theta_{B} \wedge \theta^{B}\right)\right]=-\int_{\Sigma} *(d M \wedge b) \wedge \theta^{B} \wedge * d \theta_{B} .
$$

This means that the terms independent of $p_{A}$ read

$$
\begin{aligned}
& \left\{R_{1}(b), S_{3}(M)\right\}-\left\{R_{2}(b), S_{2}(M)\right\} \\
& =-\int_{\Sigma} M b \wedge\left[\theta^{A} \wedge * d \theta^{B} *\left(d \theta_{A} \wedge \theta_{B}\right)-\theta^{A} \wedge * d \theta_{A} *\left(d \theta_{B} \wedge \theta^{B}\right)\right. \\
& \left.\quad-\theta^{A} \wedge *\left(\theta^{B} \wedge d *\left(d \theta_{B} \wedge \theta_{A}\right)\right)-* d *\left(d \theta_{A} \wedge \theta^{A}\right)\right]-\int_{\Sigma} *(d M \wedge b) \wedge \theta^{A} \wedge * d \theta_{A} .
\end{aligned}
$$

\subsubsection{Isolating constraints}

Our goal now is to express the bracket

$$
\begin{aligned}
\{R(b), S(M)\}= & \text { the terms (3.56) quadratic in } p_{A} \\
& + \text { the terms(3.73) linear in } p_{A} \\
& + \text { the terms (3.74) independent of } p_{A}
\end{aligned}
$$

as a sum of the constraints (2.5)-(2.8) smeared with some fields.

Terms quadratic in $p_{A}$ We are going to transform the last term of (3.69) to a form containing the factor $\theta^{A} \wedge * p_{A}$ being a part of the constraint $R(b)$ : applying (3.60) to the term with $\alpha^{A}=* p^{A}$ we obtain

$$
\begin{aligned}
& \left(M *\left(p_{B} \wedge \theta^{A}\right) \wedge b \wedge \theta^{B}\right) \wedge * p_{A} \\
& \left.=\theta_{A} \wedge \vec{\theta}^{B}\right\lrcorner * p^{A} \wedge\left(M *\left(p_{C} \wedge \theta_{B}\right) \wedge b \wedge \theta^{C}\right) \\
& \left.\quad-\theta_{A} \wedge * p^{A} \wedge \vec{\theta}^{B}\right\lrcorner\left(M *\left(p_{C} \wedge \theta_{B}\right) \wedge b \wedge \theta^{C}\right) .
\end{aligned}
$$

The first term at the r.h.s. above is zero-indeed, using (3.6) we get

$$
\begin{aligned}
\theta_{A} & \left.\wedge \vec{\theta}^{B}\right\lrcorner * p^{A} \wedge\left(M *\left(p_{C} \wedge \theta_{B}\right) \wedge b \wedge \theta^{C}\right) \\
& =-M *\left(p_{A} \wedge \theta^{B}\right) *\left(p_{C} \wedge \theta_{B}\right) \theta^{A} \wedge \theta^{C} \wedge b .
\end{aligned}
$$


Note now that $*\left(p_{A} \wedge \theta^{B}\right) *\left(p_{C} \wedge \theta_{B}\right)$ is symmetric in $A$ and $C$, while $\theta^{A} \wedge \theta^{C}$ antisymmetric. Transforming the remaining term in (3.75) we obtain

$$
\begin{aligned}
& \left(M *\left(p_{B} \wedge \theta^{A}\right) \wedge b \wedge \theta^{B}\right) \wedge * p_{A} \\
& \left.\left.=-M \theta_{A} \wedge * p^{A} \wedge\left(*\left(p_{C} \wedge \theta_{B} \vec{\theta}^{B}\right\lrcorner b\right) \wedge \theta^{C}-*\left(p_{C} \wedge \theta^{B}\right) b \vec{\theta}^{B}\right\lrcorner \theta^{C}\right) \\
& =-M \theta^{A} \wedge * p_{A} \wedge\left(*\left(p_{C} \wedge b\right) \theta^{C}-*\left(p_{B} \wedge \theta^{B}\right) b\right)
\end{aligned}
$$

where in the last step we used (3.8) and (3.7). Setting this result to (3.69) we arrive at an alternative description of the terms quadratic in $p_{A}$ :

$$
\begin{aligned}
& -R\left(M\left[\theta^{B} *\left(p_{B} \wedge b\right)-\frac{1}{2} b *\left(p_{B} \wedge \theta^{B}\right)\right]\right)-\int_{\Sigma} M\left[\theta^{B} *\left(p_{B} \wedge b\right)\right. \\
& \left.-\frac{1}{2} b *\left(p_{B} \wedge \theta^{B}\right)\right] \wedge \theta^{A} \wedge d \xi_{A} .
\end{aligned}
$$

Consequently, the bracket $\{R(b), S(M)\}$ is of the following form

$$
\begin{aligned}
\{R(b), S(M)\}= & -R\left(M\left[\theta^{B} *\left(p_{B} \wedge b\right)-\frac{1}{2} b *\left(p_{B} \wedge \theta^{B}\right)\right]\right) \\
& + \text { terms linear in } p_{A} \\
& + \text { the terms (3.74) independent of } p_{A},
\end{aligned}
$$

where now the phrase "terms linear in $p_{A}$ " means the terms (3.73) and the last term in (3.76). Now we are going to isolate constraints from the linear terms.

Terms linear in $p_{A}$ The terms read

$$
\begin{aligned}
& \left.\int_{\Sigma}-d M \wedge b \wedge *\left(\xi^{A} p_{A}\right)+M b \wedge d \xi^{A} \wedge * p_{A}-M \vec{\theta}_{A}\right\lrcorner p_{B} \wedge *\left(b \wedge \theta^{B}\right) \wedge d \xi^{A} \\
& \quad+M b \wedge \theta^{A} \wedge d \xi_{A} *\left(p_{B} \wedge \theta^{B}\right)-M \theta^{B} \wedge \theta^{A} \wedge d \xi_{A} *\left(p_{B} \wedge b\right)
\end{aligned}
$$

The first term can be written as

$$
\int_{\Sigma}-d M \wedge b \wedge *\left(\xi^{A} p_{A}\right)=-B(*(d M \wedge b))+\int_{\Sigma} *(d M \wedge b) \wedge \theta^{A} \wedge * d \theta_{A} .
$$

Transformation of the remaining terms in (3.78) (i.e. those which do not contain $d M$ ) to an appropriate form takes more effort. Applying (3.60) to the first of them by setting 
$\alpha^{A}=b \wedge d \xi^{A}$ and $\beta_{A}=* p_{A}$ we obtain

$$
\begin{aligned}
M b \wedge d \xi^{A} \wedge * p_{A}= & \left.\left.M \theta_{A} \wedge \vec{\theta}^{B}\right\lrcorner\left(b \wedge d \xi^{A}\right) \wedge * p_{B}+M \theta_{A} \wedge b \wedge d \xi^{A} \vec{\theta}^{B}\right\lrcorner * p_{B} \\
= & \left.\left.M \theta_{A} \wedge d \xi^{A} \wedge * p_{B} \vec{\theta}^{B}\right\lrcorner b-M \theta_{A} \wedge b \wedge * p_{B} \vec{\theta}^{B}\right\lrcorner d \xi^{A} \\
& -M b \wedge \theta_{A} \wedge d \xi^{A} *\left(p_{B} \wedge \theta^{B}\right),
\end{aligned}
$$

where in the last step we used (3.6). The last term of (3.78)

$$
\begin{aligned}
-M \theta^{B} \wedge \theta^{A} \wedge d \xi_{A} *\left(p_{B} \wedge b\right)= & -M \theta^{A} \wedge d \xi_{A} \wedge * *\left[*\left(p_{B} \wedge b\right) \wedge \theta^{B}\right] \\
= & \left.-M \theta^{A} \wedge d \xi_{A} \wedge *\left[\vec{\theta}^{B}\right\lrcorner\left(p_{B} \wedge b\right)\right] \\
= & \left.-M \theta^{A} \wedge d \xi_{A} \wedge *\left[\left(\vec{\theta}^{B}\right\lrcorner p_{B}\right) \wedge b\right] \\
& \left.-M \theta^{A} \wedge d \xi_{A} \wedge * p_{B} \vec{\theta}^{B}\right\lrcorner b
\end{aligned}
$$

-here in the second step we used (3.5). The last two results allow us to express in a more simpler form the sum of the terms in (3.78) which do not contain $d M$ :

$$
\begin{aligned}
-M \theta_{A} \wedge b & \left.\left.\wedge * p_{B} \vec{\theta}^{B}\right\lrcorner d \xi^{A}-M \vec{\theta}_{A}\right\lrcorner p_{B} \wedge *\left(b \wedge \theta^{B}\right) \wedge d \xi^{A} \\
-M \theta^{A} & \left.\wedge d \xi_{A} \wedge *\left[\left(\vec{\theta}^{B}\right\lrcorner p_{B}\right) \wedge b\right] .
\end{aligned}
$$

Our goal now is to rewrite the sum above in a form of a single term containing the factor $\theta^{A} \wedge * p_{A}$. Let us begin with the first term in (3.80):

$$
\left.\left.-M \theta_{A} \wedge b \wedge * p_{B} \vec{\theta}^{B}\right\lrcorner d \xi^{A}=M\left(*\left(b \wedge \theta_{C}\right) \vec{\theta}^{A}\right\lrcorner d \xi^{C}\right) \wedge p_{A}
$$

Setting in (3.60) $\left.\alpha^{A}=*\left(b \wedge \theta_{C}\right) \vec{\theta}^{A}\right\lrcorner d \xi^{C}$ and $\beta_{A}=p_{A}$ we obtain

$$
\begin{aligned}
\left.-M \theta_{A} \wedge b \wedge * p_{B} \vec{\theta}^{B}\right\lrcorner d \xi^{A}= & \left.\left.M \theta_{A} \wedge \vec{\theta}_{B}\right\lrcorner\left(*\left(b \wedge \theta_{C}\right) \vec{\theta}^{A}\right\lrcorner d \xi^{C}\right) \wedge p_{B} \\
& \left.\left.-M \theta_{A}\left(*\left(b \wedge \theta_{C}\right) \vec{\theta}^{A}\right\lrcorner d \xi^{C}\right) \wedge \vec{\theta}^{B}\right\lrcorner p_{B} \\
= & M d \xi^{C} \wedge *\left(b \wedge \theta_{C} \wedge \theta^{B}\right) \wedge p_{B} \\
& +M *\left[d \xi^{C} \wedge *\left(b \wedge \theta_{C}\right)\right] \wedge \theta^{B} \wedge * p_{B},
\end{aligned}
$$

where in the last step we first applied (3.6) and (3.5) to factors containing the contraction $\left.\vec{\theta}^{B}\right\lrcorner$ and then (3.8). The second term in (3.80)

$$
\begin{aligned}
& \left.-M \vec{\theta}_{A}\right\lrcorner p_{B} \wedge *\left(b \wedge \theta^{B}\right) \wedge d \xi^{A} \\
& \left.\left.=M p_{B}\left[\vec{\theta}_{A}\right\lrcorner *\left(b \wedge \theta^{B}\right)\right] \wedge d \xi^{A}-M p_{B} \wedge *\left(b \wedge \theta^{B}\right) \vec{\theta}_{A}\right\lrcorner d \xi^{A} \\
& \left.=-M d \xi^{C} *\left(b \wedge \theta_{C} \wedge \theta^{B}\right) \wedge p_{B}-M \vec{\theta}_{C}\right\lrcorner d \xi^{C} b \wedge \theta^{B} \wedge * p_{B}
\end{aligned}
$$


(in the second step we applied (3.6)). Finally, the last term in (3.80) by virtue of (3.5) can be written as

$$
\begin{aligned}
\left.-M \theta^{A} \wedge d \xi_{A} \wedge *\left[\left(\vec{\theta}^{B}\right\lrcorner p_{B}\right) \wedge b\right] & =-M *\left(* p_{B} \wedge \theta^{B}\right) \wedge b \wedge *\left(\theta^{A} \wedge d \xi_{A}\right) \\
& =M *\left[b \wedge *\left(\theta^{A} \wedge d \xi_{A}\right)\right] \wedge \theta^{B} \wedge * p_{B} .
\end{aligned}
$$

Gathering (3.81), (3.82) and the equation above and using (3.5) we obtain the desired expression for these terms in (3.78) which do not contain $d M$ :

$$
\int_{\Sigma} M\left(*\left[b \wedge *\left(\theta^{A} \wedge d \xi_{A}\right)\right]-b *\left(d \xi_{A} \wedge * \theta^{A}\right)-*\left[d \xi_{A} \wedge *\left(\theta^{A} \wedge b\right)\right]\right) \wedge \theta^{B} \wedge * p_{B}
$$

We can now simplify the term in the big parenthesis - it is enough to use (3.63) setting $\alpha=b, \beta=d \xi_{A}$ and $\kappa=\theta^{A}$ to get

$$
\begin{aligned}
& \int_{\Sigma} M\left(-d \xi_{A} *\left(b \wedge * \theta^{A}\right)\right) \wedge \theta^{B} \wedge * p_{B} \\
& \quad=-R\left(M d \xi_{A} *\left(b \wedge * \theta^{A}\right)\right)-\int_{\Sigma} M *\left(b \wedge * \theta^{A}\right) d \xi_{A} \wedge \xi^{B} d \theta_{B}
\end{aligned}
$$

which is the final form of the terms in (3.78) which do not contain $d M$.

The Eqs. (3.80) and (3.83) allow us to express the terms linear in $p_{A}$ appearing in (3.77) as a sum of constraints and terms independent of the momenta. Consequently, (3.77) can be written in the following form:

$$
\begin{aligned}
\{R(b), S(M)\}= & -R\left(M\left[\theta^{B} *\left(p_{B} \wedge b\right)-\frac{1}{2} b *\left(p_{B} \wedge \theta^{B}\right)+d \xi_{A} *\left(b \wedge * \theta^{A}\right)\right]\right) \\
& -B(*(d M \wedge b))+\text { terms independent of } p_{A}
\end{aligned}
$$

where the phrase "terms independent of $p_{A}$ " means here the terms given by (3.74), the last term in (3.80) and the last one in (3.83).

Terms independent of $p_{A}$ Gathering all the terms under consideration which appear in (3.84) we see that the sum of the last term of (3.74) and the last term of (3.80) is zero. Note now that the last term in (3.83) contains $\xi^{A}$ which does not appear in the others term. To get rid of $\xi^{A}$ let us use (3.61):

$$
-\int_{\Sigma} M *\left(b \wedge * \theta^{A}\right) d \xi_{A} \wedge \xi^{B} d \theta_{B}=-\int_{\Sigma} M b \wedge * \theta^{A} *\left[d *\left(* \theta_{A} \wedge \theta^{B}\right) \wedge d \theta_{B}\right]
$$


Consequently, the terms in (3.84) independent of $p_{A}$ read

$$
\begin{aligned}
& -\int_{\Sigma} M b \wedge\left(\theta^{A} \wedge * d \theta^{B} *\left(d \theta_{A} \wedge \theta_{B}\right)-\theta^{A} \wedge * d \theta_{A} *\left(d \theta_{B} \wedge \theta^{B}\right)\right. \\
& -\theta^{A} \wedge *\left(\theta^{B} \wedge d *\left(d \theta_{B} \wedge \theta_{A}\right)\right)-* d *\left(d \theta_{A} \wedge \theta^{A}\right) \\
& \left.+* \theta^{A} *\left(d *\left(* \theta_{A} \wedge \theta^{B}\right) \wedge d \theta_{B}\right)\right) .
\end{aligned}
$$

By direct calculations with application of tensor calculus (Sect. 3.1.3) it is possible to show that the terms in the big parenthesis sum up to zero for every $\theta^{A}$.

Final result Thus the formula (3.84) turns into the final expression of the Poisson bracket of $R(b)$ and $S(M)$ :

$$
\begin{aligned}
\{R(b), S(M)\}= & -R\left(M\left[\theta^{B} *\left(p_{B} \wedge b\right)-\frac{1}{2} b *\left(p_{B} \wedge \theta^{B}\right)+d \xi_{A} *\left(b \wedge * \theta^{A}\right)\right]\right) \\
& -B(*(d M \wedge b)) .
\end{aligned}
$$

\subsection{Poisson bracket of $B(a)$ and $S(M)$}

Using the same procedure as in the previous subsection we arrive at

$$
\begin{aligned}
\{B(a), S(M)\}= & -B\left(M\left[\theta^{B} *\left(p_{B} \wedge a\right)-\frac{1}{2} a *\left(p_{B} \wedge \theta^{B}\right)\right]\right)+R(*(d M \wedge a)) \\
& + \text { terms linear in } p_{A}+\text { terms independent of } p_{A}
\end{aligned}
$$

where the terms linear in $p_{A}$ read

$$
\begin{aligned}
& \int_{\Sigma} M\left[\theta^{B} *\left(p_{B} \wedge a\right)-\frac{1}{2} a *\left(p_{B} \wedge \theta^{B}\right)\right] \wedge \theta^{A} \wedge * d \theta_{A}-M d *\left(* a \wedge \theta^{A}\right) \wedge p_{A} \\
& +M a \wedge \theta^{B} \wedge * d \theta_{A} *\left(p_{B} \wedge \theta^{A}\right)+M d *\left(a \wedge \theta_{A}\right) \wedge \theta^{B} *\left(p_{B} \wedge \theta^{A}\right) \\
& -M a \wedge \theta^{B} \wedge * d \theta_{B} *\left(p_{A} \wedge \theta^{A}\right)-\frac{M}{2} d *\left(a \wedge \theta_{A}\right) \wedge \theta^{A} *\left(p_{B} \wedge \theta^{B}\right) \\
& +M *\left(a \wedge \theta^{B} \wedge \theta^{A}\right) * p_{A} \wedge d \theta_{B}-M a \wedge * p_{A} \wedge \theta^{B} *\left(d \theta_{B} \wedge \theta^{A}\right)
\end{aligned}
$$

and the terms independent of $p_{A}$ read

$$
\begin{aligned}
& \int_{\Sigma} d M \wedge a \wedge *\left(\xi^{A} d \theta_{A}\right)-d M \wedge a \wedge *\left(\xi^{A} d \theta_{A}\right)-M a \wedge * d \theta_{A} \wedge d \xi^{A} \\
& +M a \wedge \theta^{B} \wedge d \xi^{A} *\left(d \theta_{B} \wedge \theta_{A}\right)-M *\left(* d \theta_{B} \wedge \theta_{A}\right) \wedge *\left(a \wedge \theta^{B}\right) \wedge d \xi^{A} \\
& +M a \wedge \theta^{B} \wedge \xi^{A} d *\left(d \theta_{B} \wedge \theta_{A}\right)-M a \wedge \xi^{A} d \theta_{B} *\left(d \theta_{A} \wedge \theta^{B}\right) \\
& +M a \wedge \xi^{A} d \theta_{A} *\left(d \theta_{B} \wedge \theta^{B}\right) .
\end{aligned}
$$


Note now that the form of constraints at the r.h.s. of (3.87) resemble closely the form of the constraints at the r.h.s. of (3.86). We then assume that

$$
\begin{aligned}
\{B(a), S(M)\}= & -B\left(M\left[\theta^{B} *\left(p_{B} \wedge a\right)-\frac{1}{2} a *\left(p_{B} \wedge \theta^{B}\right)+d \xi_{B} *\left(a \wedge * \theta^{B}\right)\right]\right) \\
& +R(*(d M \wedge a)) .
\end{aligned}
$$

To justify the assumption one can proceed as follows: one adds to the r.h.s. of (3.87) zero expressed as

$$
\begin{aligned}
0= & B\left(M d \xi_{B} *\left(a \wedge * \theta^{B}\right)\right)+B\left(M d \xi_{B} *\left(a \wedge * \theta^{B}\right)\right) \\
= & -B\left(M d \xi_{B} *\left(a \wedge * \theta^{B}\right)\right)+\int_{\Sigma} M d \xi_{B} *\left(a \wedge * \theta^{B}\right) \wedge\left(\theta^{A} \wedge * d \theta_{A}+\xi^{A} p_{A}\right) \\
= & -B\left(M d \xi_{B} *\left(a \wedge * \theta^{B}\right)\right)+\int_{\Sigma} M d \xi_{B} *\left(a \wedge * \theta^{B}\right) \theta^{A} \wedge * d \theta_{A} \\
& +M d *\left(* \theta_{B} \wedge \theta^{A}\right) \wedge p_{A} *\left(a \wedge * \theta^{B}\right)
\end{aligned}
$$

(here in the last step we used (3.61)). Next using tensor calculus (Sect. 3.1.3) it is possible to show that all the remaining terms linear in $p_{A}$ sum up to zero, and that similarly do all the remaining terms independent of the momenta. Note that now the description of the terms linear in and independent of the momenta given just below Eq. (3.87) has to be completed by taking into account the two last term in (3.89).

\subsection{Poisson brackets of $V(\vec{M})$}

The functional derivatives of the smeared vector constraint $V(\vec{M})$ (see (2.8)) are of the following form [9]:

$$
\frac{\delta V(\vec{M})}{\delta \theta^{A}}=-\mathcal{L}_{\vec{M}} p_{A}, \quad \frac{\delta V(\vec{M})}{\delta p_{A}}=\mathcal{L}_{\vec{M}} \theta^{A}
$$

This means that gauge transformations defined by the constraint $V(\vec{M})$ act on the canonical variables as pull-back given by diffeomorphisms $\left\{\chi_{\lambda}\right\}_{\lambda \in \mathbb{R}}$ on $\Sigma$ - the diffeomorphisms form a one-parameter group generating the vector field $\vec{M}$. Consequently, one can expect that if $C(T)$ is a constraint on the phase space smeared with a tensor field $T$ independent of the canonical variables then

$$
\{V(\vec{M}), C(T)\}=C\left(\mathcal{L}_{\vec{M}} T\right)
$$


where $\mathcal{L}_{\vec{M}} T$ is a Lie derivative of $T$ with respect to $\vec{M}$. Indeed, it was shown in [9] that

$$
\left\{V(\vec{M}), V\left(\vec{M}^{\prime}\right)\right\}=V\left(\left[\vec{M}, \vec{M}^{\prime}\right]\right)=V\left(\mathcal{L}_{\vec{M}} \vec{M}^{\prime}\right)
$$

where $\left[\vec{M}, \vec{M}^{\prime}\right]$ is the Lie bracket of the vector fields $\vec{M}, \vec{M}^{\prime}$ on $\Sigma$. Using the formula [9]:

$$
\mathcal{L}_{\vec{M}}(\alpha \wedge * \beta)=\mathcal{L}_{\vec{M}} \alpha \wedge * \beta+\alpha \wedge * \mathcal{L}_{\vec{M}} \beta+\mathcal{L}_{\vec{M}} \theta^{A} \wedge\left(\alpha *_{A}^{\prime} \beta\right)
$$

and the following well known properties of the Lie derivative:

$$
0=\int_{\Sigma}\left(\mathcal{L}_{\vec{M}} \alpha\right) \wedge \beta+\int_{\Sigma} \alpha \wedge \mathcal{L}_{\vec{M}} \beta, \quad d\left(\mathcal{L}_{\vec{M}} \gamma\right)=\mathcal{L}_{\vec{M}}(d \gamma)
$$

where $\alpha \wedge \beta$ is a three-form, and $\gamma$ is any $k$-form on $\Sigma$ it is not difficult to show that the remaining constraints $S(M), B(a)$ and $R(b)$ satisfy (3.91).

\section{Summary}

To summarize the calculations let us list the results. The Poisson brackets of boost and rotation constraints (Eqs. (3.24), (3.23) and (3.25)):

$$
\begin{aligned}
\left\{B(a), B\left(a^{\prime}\right)\right\} & =-R\left(*\left(a \wedge a^{\prime}\right)\right), \\
\left\{R(b), R\left(b^{\prime}\right)\right\} & =R\left(*\left(b \wedge b^{\prime}\right)\right), \\
\{B(a), R(b)\} & =B(*(a \wedge b)) .
\end{aligned}
$$

The bracket of the scalar constraints (Eq. (3.55)):

$$
\begin{aligned}
\left\{S(M), S\left(M^{\prime}\right)\right\}= & V(\vec{m})+B\left(\theta^{B} *\left(m \wedge p_{B}\right)-\frac{1}{2} *\left(m \wedge \xi^{B} * d \theta_{B}\right)\right. \\
& -*\left[m \wedge *\left(\theta^{B} \wedge * p_{B}\right)\right]-\frac{1}{2} *\left(* m \wedge \theta^{B}\right) * p_{B} \\
& \left.+\frac{1}{2} *\left[*\left(m \wedge \theta^{B}\right) \wedge * p_{B}\right]\right)+R\left(-\theta^{B} *\left(m \wedge d \theta_{B}\right)\right. \\
& -\frac{1}{2} *\left(m \wedge \xi^{B} * p_{B}\right)+*\left[m \wedge *\left(\theta^{B} \wedge * d \theta_{B}\right)\right] \\
& \left.+\frac{1}{2} *\left(* m \wedge \theta^{B}\right) * d \theta_{B}-\frac{1}{2} *\left[*\left(m \wedge \theta^{B}\right) \wedge * d \theta_{B}\right]\right) .
\end{aligned}
$$

In this formula

$$
m:=M d M^{\prime}-M^{\prime} d M .
$$


The brackets of the boost and rotation constraints and the scalar one (Eqs. (3.88) and (3.86)):

$$
\begin{aligned}
\{B(a), S(M)\}= & -B\left(M\left[\theta^{B} *\left(p_{B} \wedge a\right)-\frac{1}{2} a *\left(p_{B} \wedge \theta^{B}\right)+d \xi_{B} *\left(a \wedge * \theta^{B}\right)\right]\right) \\
& +R(*(d M \wedge a)) \\
\{R(b), S(M)\}= & -R\left(M\left[\theta^{B} *\left(p_{B} \wedge b\right)-\frac{1}{2} b *\left(p_{B} \wedge \theta^{B}\right)+d \xi_{A} *\left(b \wedge * \theta^{A}\right)\right]\right) \\
& -B(*(d M \wedge b)) .
\end{aligned}
$$

The brackets of the vector constraint (Eq. (3.91)):

$$
\begin{gathered}
\left.\left\{V(\vec{M}), V\left(\vec{M}^{\prime}\right)\right\}=V\left(\mathcal{L}_{\vec{M}} \vec{M}^{\prime}\right), \quad V(\vec{M}), S(M)\right\}=S\left(\mathcal{L}_{\vec{M}} M\right), \\
\{V(\vec{M}), B(a)\}=B\left(\mathcal{L}_{\vec{M}} a\right), \quad\{V(\vec{M}), R(b)\}=R\left(\mathcal{L}_{\vec{M}} b\right) .
\end{gathered}
$$

In the formulae above $\mathcal{L}_{\vec{M}}$ denotes the Lie derivative on $\Sigma$ with respect to the vector field $\vec{M}$.

As mentioned in the introduction a discussion of the results can be found in [1], here we restrict ourselves to a statement that a Poisson bracket of every pair of the constraints (2.5) - (2.8) is a sum of the constraints smeared with some fields. In other words, the constraint algebra presented above is closed.

Acknowledgments I am grateful to Jędrzej Świeżewski for his cooperation in the research on a Hamiltonian model described in [9] which was for me a preparatory exercise for deriving the results described in this paper. I am also grateful to Jerzy Lewandowski for a valuable discussion.

Open Access This article is distributed under the terms of the Creative Commons Attribution License which permits any use, distribution, and reproduction in any medium, provided the original author(s) and the source are credited.

\section{References}

1. Okołów, A.: ADM-like Hamiltonian Formulation of Gravity in the Teleparallel Geometry. Gen. Relativ. Gravit. 45, 2569-2610. E-print arXiv:1111.5498. (2013)

2. Okołów, A.: Variables Suitable for Constructing Quantum States for the Teleparallel Equivalent of General Relativity I. Gen. Relativ. Gravit. E-print arXiv:1305.4526. (2013) (accepted)

3. Okołów, A.: Variables Suitable for Constructing Quantum States for the Teleparallel Equivalent of General Relativity II. Gen. Relativ. Gravit. E-print arXiv:1308.2104. (2013) (accepted)

4. Okołów, A.: Kinematic Quantum States for the Teleparallel Equivalent of General Relativity E-print arXiv:1304.6492. (2013)

5. Maluf, J.W., da Rocha-Neto, J.F.: Hamiltonian formulation of general relativity in the teleparallel geometry. Phys. Rev. D 64, 084014. E-print arXiv:gr-qc/0002059. (2001)

6. da Rocha-Neto, J.F., Maluf, J.W., Ulhoa, S.C.: Hamiltonian formulation of unimodular gravity in the teleparallel geometry. Phys. Rev. D 82, 124035. E-print arXiv:1101.2425. (2010)

7. Blagojević, M., Nikolić, I.A.: Hamiltonian structure of the teleparallel formulation of GR. Phys. Rev. D 62, 024021. E-print arXiv:hep-th/0002022. (2000)

8. Mielke, E.W.: Ashtekar's complex variables in general relativity and its teleparallelism equivalent. Ann. Phys. 219, 78-108 (1992) 
9. Okołów, A., Świeżewski, J.: Hamiltonian formulation of a simple theory of the teleparallel geometry Class. Quant. Grav. 29, 045008. E-print arXiv:1111.5490. (2012)

10. Nester, J.M.: Positive energy via the teleparallel Hamiltonian. Int. J. Mod. Phys. A 4, 1755-1772 (1989) 